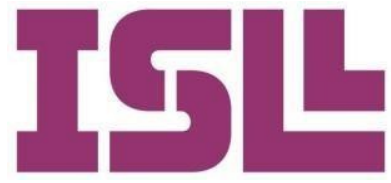

Número 12

Diciembre 2019

\title{
Comprensión lectora y el rendimiento académico en Educación Primaria
}

\author{
Efrén Viramontes Anaya \\ https://orcid.org/0000-0003-4378-3095 \\ Anahí Amparán Muro \\ https://orcid.org/0000-0003-0390-0883 \\ Luz Divina Núñez \\ Sifuentes \\ Escuela Normal Rural Ricardo Flores Magón
}

Recibido: 26-04-2018

Aceptado: 29-11-2019

Pág. 65 a la 82

\section{Palabras clave:}

Comprensión lectora, rendimiento académico, lectura, fluidez de lectura, velocidad de lectura.

\section{Resumen:}

Esta investigación aborda el tema de la cuantitativo, la entrevista y la observación comprensión lectora y la relación que ayudaron a interpretar los niveles de existe con el rendimiento académico en lectura de los alumnos y los tipos de tercer grado de educación primaria. El desaciertos en la misma; se realizó un propósito es la valoración de los nexos análisis descriptivo de las variables, así que tienen ambas variables y tomar como del cálculo de correlaciones Rho de conciencia de la importancia de la Spearman y t de student para muestras lectura en los procesos académicos en independientes. Los resultados indican que general. El estudio se desarrolló con la comprensión se correlaciona niños de dos grupos de tercer grado de significativamente con el una escuela primaria mexicana, cuyas aprovechamiento académico y que la edades se encuentran entre los ocho y condición de ser hombre o mujer no está nueve años, el nivel socioeconómico es asociada con los niveles de comprensión medio y el contexto urbano pequeño, de la lectura, aunque se muestran enclavado en un área rural. La coincidencias con otras investigaciones metodología se hizo bajo un enfoque que las mujeres presentan niveles ligeramente más altos. 


\section{Introducción}

La investigación se desarrolló en una escuela del norte de México, en la cabecera de un municipio rural; se realizó en dos grupos de tercer grado de educación primaria: el primero de 26 alumnos, 16 hombres y 10 mujeres; el segundo de 28 alumnos, 13 niñas y 15 niños. Todos los sujetos de investigación con edades entre los 8 y los 9 años. La escuela se encuentra en el centro de la población de 16000 habitantes aproximadamente, rodeada por pequeñas comunidades rurales que confluyen en esta mancha semiurbana para asuntos administrativos del municipio, para acudir a las diferentes escuelas o para adquirir artículos que necesitan para sus viviendas y alimentación. Esta comunidad es deficiente desde el punto de vista alfabetizador, toda vez que no existen portadores de textos en la cantidad que puede haber en una gran ciudad, ni los suficientes actos de lectura en sus hogares, como para que tuviera un buen impacto en la alfabetización de los niños.

La justificación para el abordaje de esta temática de investigación se centra en los resultados de exámenes de organismos nacionales e internacionales sobre la comprensión lectora de los niños en las escuelas mexicanas, así como en las evaluaciones realizadas en el ámbito escolar local. A esto se le agrega la importancia que tiene la lectura como herramienta para el análisis cognitivo y el aprendizaje de los contenidos escolares y no escolares que impactan en la formación general de la persona.

En las últimas décadas se ha evaluado muy puntualmente la comprensión lectora en las escuelas de la mayoría de los países del mundo ya sea por organismos internos o externos a cada país; derivado de estas evaluaciones se ha considerado a la lectura como un asunto prioritario de atención, principalmente en las escuelas de educación básica. En México, los resultados 2012 del Programme for International Student Assessment (PISA), (OCDE, 2012) han sido muy bajos, con respecto a los demás países pertenecientes a la OCDE, remontar esos resultados no es tan sencillo, toda vez que se establece en ese informe que "de mantenerse las tasas de mejora actuales, a México le tomará más de 25 años para alcanzar los niveles promedio actuales de la OCDE en matemáticas y más de 65 años en lectura" (OCDE, 2012, p. 3). Estos resultados son ponderados en una justa dimensión por Silva (2014) al señalar que no son simples cifras, sino que son reflejo de las realidades educativas de los países evaluados, y que implican un problema educativo real que requiere ser atendido.

En 2017 el INEE (2018) aplicó el examen denominado PLANEA-ELSEN (Plan Nacional para la Evaluación de los Aprendizajes - Evaluación del Logro referida al Sistema Educativo Nacional), evaluando el desarrollo del lenguaje y la comunicación, entre otros rubros, cuyos resultados establecen que existe un $33.8 \%$ de los estudiantes de secundaria que se encuentran en nivel deficiente, mientras que sólo el $26.1 \%$ de la población evaluada resultó con puntajes de niveles satisfactorio y sobresaliente. En estos resultados, que se muestran los niveles más bajos y más altos, se percibe, además, una clara tendencia hacia la insuficiencia de elementos necesarios en el desarrollo del lenguaje y la comunicación, en esto, la comprensión de la lectura juega un papel fundamental.

Después de analizar los resultados nacionales e internacionales sobre la evaluación de la lectura, se considera que este tema es crucial para formar a los ciudadanos en el ámbito de la literacidad, en el que la escritura y la comprensión lectora son elementos fundamentales. El desarrollo de la literacidad impacta en el ámbito escolar como en la vida diaria del sujeto, es por ello que existe la necesidad de mejorar la lectura de los niños desde temprana edad. 
Por esta razón se requiere establecer formas de evaluación muy puntuales que puedan ser aplicadas por todos los docentes de la educación básica. Se parte del supuesto de que la comprensión lectora es la parte esencial del desarrollo académico de los estudiantes, toda vez que se convierte en la herramienta principal de aprendizaje como lo plantean Solé (2012) y García, Arévalo y Hernández (2017, p. 158), estos últimos lo expresan de manera textual así: "la lectura en contextos académicos ha cobrado mayor importancia y está llamada a participar cada vez más activamente de los procesos de aprendizaje continuo y aprendizaje para la vida". Se advierte, además, que el aprendizaje es afectado por múltiples factores, y que la lectura es sólo uno de ellos. Con este fundamento surge el planteamiento principal de esta investigación con la pregunta: ¿qué relación existe entre la comprensión lectora y el rendimiento académico de los alumnos de tercer grado de educación primaria?

Se pretende identificar la relación entre estas dos variables; primero, como una forma de comprobar lo que parece obvio, y también por establecer de manera tangible cómo se comportan los elementos inmiscuidos en la comprensión lectora con referencia al aprovechamiento escolar que tienen los estudiantes en la escuela primaria. Se agrega al análisis el estudio de género en los resultados de la evaluación de la lectura.

Algunas de las investigaciones que sirven como antecedente de este estudio son: el de Aguillón (2014), con respecto al análisis de los elementos léxicos, semánticos y sintácticos de la comprensión lectora en niños en 11 años, con metodología cuantitativa; el estudio que hizo Solís (2014), sobre la comprensión lectora y rendimiento académico, se analizó la correlación entre los niveles de la comprensión lectora y el rendimiento académico en el área de comunicación de los alumnos de segundo grado de educación primaria, en este estudio se llegó a la conclusión de que existe una relación significativa positiva entre la variable comprensión lectora y el rendimiento en el área de comunicación; otro estudio fue el de Ramos (2013), sobre la comprensión lectora como una herramienta básica en la enseñanza de las ciencias naturales, se propuso implementar una estrategia basada en el planteamiento de preguntas para mejorar el nivel de compresión lectora de textos científicos en el área de ciencias naturales, el resultado establece que la mayoría de los alumnos tuvieron mejoría, en comprensión literal fue donde se ubicó la mayor población; enseguida inferencial y por último crítica. Otros referentes son las aportaciones de Madero y Gómez (2011), sobre el proceso de comprensión lectora en alumnos de tercero de secundaria y de Hernández (2007) con respecto a los ritmos de aprendizaje en la comprensión lectora en alumnos de tercer grado de primaria.

Una de las definiciones de lectura que sirven de referente conceptual es la que ofrece la $\operatorname{OCDE}(2006$, p.48) que entiende la lectura como "la capacidad de comprender, utilizar y analizar textos escritos, para alcanzar los objetivos del lector, desarrollar sus conocimientos y posibilidades y participar en la sociedad", misma que es complementada con la idea de Gómez-Palomino (2011, p. 28) cuando señala que esta actividad "va más allá de la producción constructiva del texto, lo cual se logra bajo diversas condiciones endógenas y exógenas", lo que implica una acción intelectual interna del sujeto y la interacción con el texto, que se encuentra fuera de él, con información específica, temática contextualizada, expresada mediante signos determinados. En resumen, se plantea la lectura como una acción comunicativa entre el escritor y el lector. La acción interna implica al ámbito cognitivo, desde la decodificación, la identificación de las palabras en el texto, el significado semántico, así como la asignación mental comprensiva proveniente de experiencia contextualizada del sujeto lector. 
En lo que corresponde a la comprensión lectora, Gómez-Palomino (2011, p. 30) la explica como "actividad constructiva compleja de carácter estratégico que implica las interacciones de características del lector y del texto, dentro de un contexto determinado", asignando al sujeto un rol muy importante, toda vez que tiene que construir el significado del texto; entre la información visual y no visual planteada por Smith (1989), entre el texto y el marco de referencia del sujeto. Con esto se establece un patrón de comprensión semántico que da sentido a lo que se lee, implicando una serie de acciones intelectuales, o estrategias, propuestas por Goodman (1996), que el lector pone en práctica: muestreo, predicción, inferencia, confirmación y autocorrección.

La intención con la que se aproxima el lector al texto es un factor muy importante para la comprensión, toda vez que el motivo actúa como un filtro de información, en la que se pondera lo que se busca y desecha lo intranscendente para este propósito.

La Secretaría de educación pública de México (SEP, 2011) divide los aspectos que se deben alcanzar en cuanto a comprensión lectora en ciclos, en este caso los que intervienen en esta investigación directamente son los del segundo ciclo que involucra a tercer y cuarto grado de educación primaria, los cuales son: Identificar la idea principal de un texto, localizar la información específica de un texto y utiliza información contenida en un texto para desarrollar un argumento.

Para clasificar el nivel en que se encuentran en cada aspecto, la SEP (2011) propone cuatro niveles de logro, los cuales se utilizan en la educación básica, por este motivo los rasgos de cada nivel se pueden adaptar al grado en el que se utiliza. Los niveles establecidos son: requiere apoyo, se acerca al estándar, estándar y avanzado.

En cuanto a los tipos de comprensión lectora se tomaron de referencia los que propone Pinzás (1997): nivel de decodificación, comprensión literal, comprensión inferencial y comprensión crítica.

Se consideró también a la fluidez lectora como parte de la comprensión, porque se refiere a las habilidades que tiene el alumno para leer el texto de manera que acceda a la información y la procese, respetando todos los signos de puntuación como elementos paralingüísticos que aportan contenido (SEP, 2011). Con respecto al constructo fluidez lectora, Fumagalli, Barreyro y Jaichenco (2017) explican que cuando se lee se hace con un ritmo y velocidad específico que le permita al lector ir reconstruyendo el contenido del texto, y que cuando, por cualquier motivo, la lectura pierde ese ritmo, la reconstrucción de significados se interrumpe o se pierde. Esto depende, en gran medida, de un proceso que, según estos autores, se caracteriza por lo que denominan estrategias de decodificación y reconocimiento automático de palabras. Ellos, al igual que Hudson, Torgesen, Lane y Turner (2012), consideran como parte de la fluidez a la velocidad, la precisión y otros aspectos como la decodificación y la combinación fonémica.

Por su parte la SEP (2011) propone cuatro niveles para evaluar la fluidez lectora: 1) requieren apoyo, 2) se acercan al estándar, 3) estándar y 4) avanzado (tabla 1), este último nivel implica la lectura de textos completos, con signos de puntuación y entonación adecuada, con capacidad de comprender el sentido del texto, y actuar en consecuencia o proponer algo a partir él. Esta propuesta de evaluación es la que se utilizó en esta investigación. 
Tabla 1: Niveles de velocidad lectora

\begin{tabular}{|c|c|c|c|c|}
\hline \multicolumn{5}{|c|}{ Primaria } \\
\hline Grado Escolar & $\begin{array}{ll}\text { Nivel } & \text { Requiere } \\
\text { Apoyo } & \\
\end{array}$ & $\begin{array}{l}\text { Nivel Se acerca al } \\
\text { estándar }\end{array}$ & Estándar & $\begin{array}{l}\text { Nivel } \\
\text { Avanzado }\end{array}$ \\
\hline Primero & Menor que 15 & De 15 a 34 & De 35 a 59 & Mayor que 59 \\
\hline Segundo & Menor que 35 & De 35 a 59 & De 60 a 84 & Mayor que 84 \\
\hline Tercero & Menor que 60 & De 60 a 84 & De 85 a 99 & Mayor que 99 \\
\hline Cuarto & Menor que 85 & De 85 a 99 & De 100 a 114 & Mayor que 114 \\
\hline Quinto & Menor que 100 & De 100 a 114 & De 115 a 124 & Mayor que 124 \\
\hline Sexto & Menor que 115 & De 115 a 124 & De 125 a 134 & Mayor que 134 \\
\hline
\end{tabular}

Fuente: SEP, 2012, p.11

La velocidad lectora, que también interviene en la comprensión, se considera como la cantidad de palabras que se pueden leer en un tiempo determinado, se evalúa de manera independiente, aunque está ligada a la fluidez. Una velocidad demasiado lenta generalmente impide comprender, mientras que una excesiva velocidad haría menos posible dicha comprensión, estos límites dependen de cada sujeto, sus habilidades lectoras, del texto y de su marco de referencia temática con el que hace frente a la lectura.

La medición de este atributo de la lectura se hace directamente considerando sólo las palabras por minuto que se leen por una persona, como se plantea en SEP (2011). El hecho de tomarla en cuenta para realizar una medición de la comprensión, se hace sólo para efectos de complementar la valoración de la variable denominada comprensión lectora integral, y por atender la sugerencia oficial de evaluación de la Secretaría de educación pública, no como un reflejo de postura teórica que establezca a la velocidad de la lectura como elemento independiente de la fluidez, ya que se coincide más con la idea de considerar a la velocidad como parte inherente a la fluidez, como lo proponen Hudson, et al (2012).

El proceso de la comprensión lectora no es lineal, por tanto, requiere tomar en cuenta otros elementos además de la velocidad de la lectura, como el hecho de realizar regresiones en la lectura y aplicar autocorrecciones, esto hace que tarde más tiempo para leer, pero no significa que comprenda menos. Por ello su valoración debe incluir más elementos a fin de tener una idea más integral de su proceso, como los que se incluyen en la tabla 2.

Para efectos de esta investigación se considera a la evaluación de la comprensión lectora como lo plantea Casanova (1998, p. 71): “obtención de información rigurosa y sistemática para contar con datos válidos y fiables cerca de una situación con objeto de formar y emitir un juicio de valor con respecto a ella", con esto se refiere a que debe servir para mejorar la práctica educativa, así como para verificar en qué aspectos se puede optimizar los procesos de aprendizaje. Se constituye en un referente para la toma de decisiones de intervención en el ámbito educativo.

También es importante considerar, como señalan Gutiérrez-Braojos y Salmerón (2012), que para realizar la evaluación lectora se recomienda el uso diversificado de técnicas de recogida de información para valorar la comprensión. Esto posibilita la realización de una triangulación de información que permite mayor precisión en la interpretación clínica y/o cuantitativa de los avances o niveles en la comprensión de los textos escritos.

Se coincide con el supuesto de que los enfoques de enseñanza del lenguaje tienen una influencia muy marcada en la calidad de la comprensión lectora de los estudiantes, sobre esto Solé 
(2007) explica dos procesos didácticos en la construcción de la alfabetización inicial de los niños, que afectan la manera en que se desarrolla la comprensión lectora en la etapa temprana de los mismos; el primer modelo, lo denomina bottom up, es un método ascendente de lectura, en éste se considera que el lector primero analiza las letras, enseguida las palabras para así crear un esquema que le ayude a comprender el texto. Se considera ascendente porque va de lo más simple a lo más complejo que es la comprensión.

Al respecto, Condemarín (1989, p. 75) señala que "las destrezas más globales son la identificación de la palabra y la comprensión, las cuales se dividen en unidades disgregadas de contenidos y se asocian a respuestas simples o bien, a clases de respuestas complejas, automáticas y precisas", reflexión que coincide con lo planteado por Solé (2007) en el modelo top down, descendente $u$ holístico. En éste se plantea el texto de manera global y contextualizada, se genera una idea integral de lo que se piensa tratar, para después proceder a la lectura y al análisis más específico del contenido, este proceso se considera más adecuado que el primero, porque se realizan acciones cognitivas y se aplica el conocimiento que ya se posee, se contextualiza el escrito haciendo conexiones significativas con el texto. Estas dos formas de proceder se definen en Llorens (2015) como procedimientos analíticos y sintéticos. Existe un tercer modelo, más efectivo, nombrado por Jiménez (2014) como interactivo, en el que se realizan acciones complementarias entre los procesos analíticos (top down) y sintéticos (bottom up), este enfoque es el que se toma como supuesto para desarrollar comprensión de calidad en esta investigación, basando la interacción en las prácticas sociales del lenguaje, es decir, considerando a la lectura dentro de una situación comunicativa con sentido y función real.

En lo que respecta a la relación existente entre el sexo o género de los estudiantes y la posibilidad de que los hombres o las mujeres sean o no más proclives a tener mejor comprensión lectora, se encontró evidencia investigativa, Ostria, López y Valenzuela (2014) y el Instituto Nacional para la Evaluación Educativa (INEE, 2018), que señala que las mujeres presentan mejores niveles de comprensión que los hombres. Este asunto de género se aborda en este estudio para aportar más información al respecto, toda vez que la prueba de esta hipótesis aporta elementos para que los docentes la consideren en sus estrategias didácticas, con intervenciones específicas necesarias.

La atención didáctica para el desarrollo de la comprensión lectora en los niños de educación básica, es apoyada por las aportaciones científicas de Viana, Ribeiro y Santos (2014) y del INEE (2012), los primeros en el sentido y propósito que debe tener la evaluación de la comprensión, para la mejorar los procesos educativos de los estudiantes; el segundo, respecto a las consideraciones concretas de la enseñanza en el tema de la lectura y las posibilidades que se tienen para avanzar en la comprensión.

Se establece que la variable dependiente de esta investigación, el rendimiento escolar, es un factor que está inserto en la educación, que se mide con la suma de las calificaciones en las que intervienen diferentes factores que determinan los resultados de los estudiantes, y se define como: "los logros cuantificados que se alcanzan en ciertos aprendizajes o asignaturas según el plan de estudio" (Garbanzo, 2007, p. 46).

\section{Objetivos e hipótesis}

Los objetivos principales de la investigación son dos: el primero, analizar la relación entre la comprensión lectora e indicadores de lectura (fluidez, velocidad, y localización de información) y el rendimiento académico de los alumnos; el segundo, establecer si existen diferencias entre el sexo de los alumnos/as y los mismos indicadores de la lectura antes descritos. 
Las hipótesis sobre correlación Rho de Spearman que se sometieron a prueba son la versión nula (análisis bilateral) de las siguientes hipótesis de investigación:

$\mathrm{H}_{1}$ : Existe relación entre la fluidez de lectura y el rendimiento académico de los alumnos de tercer grado

$\mathrm{H}_{2}=$ Existe relación entre la velocidad de la lectura y el rendimiento académico de los alumnos de tercer grado

$\mathrm{H}_{3}$ : Existe relación entre la identificación de la idea principal de un texto y el rendimiento académico de los alumnos de tercer grado

$\mathrm{H}_{4}$ : Existe relación entre la localización de la información específica de un texto, tabla o gráfica y el rendimiento académico de los alumnos de tercer grado

$\mathrm{H}_{5:}$ Existe relación entre la utilización información contenida en un texto para desarrollar un argumento y el rendimiento académico de los alumnos de tercer grado

$\mathrm{H}_{6:}$ Existe relación entre la comprensión lectora integral y el rendimiento académico de los alumnos de tercer grado

También se realizó el análisis de $t$ de student para muestras independientes de la variable sexo, en relación con los puntajes de las variables que representan a la comprensión lectora integral y sus aspectos específicos. La regla de decisión es similar a la de las pruebas de correlación $(\mathrm{p}=.05)$.

En el análisis de $t$ de student para muestras independientes que se sometieron a prueba la versión nula (análisis bilateral) de las siguientes hipótesis de investigación:

$\mathrm{H}_{1}$ : Existen diferencias significativas entre el sexo de los alumnos de tercer grado y fluidez de la lectura.

$\mathrm{H}_{2}$ : Existen diferencias significativas entre el sexo de los alumnos de tercer grado y velocidad de la lectura.

$\mathrm{H}_{3}$ : Existen diferencias significativas entre el sexo de los alumnos de tercer grado y identificación de la idea principal de un texto.

$\mathrm{H}_{4}$ : Existen diferencias significativas entre el sexo de los alumnos de tercer grado y localización de la información específica de un texto, tabla o gráfica.

$\mathrm{H}_{5}$ : Existen diferencias significativas entre el sexo de los alumnos de tercer grado y utiliza información contenida en un texto para desarrollar un argumento.

$\mathrm{H}_{6}$ : Existen diferencias significativas entre el sexo de los alumnos de tercer grado y comprensión de lectura integral.

\section{Método}

\section{Participantes}

La investigación se realizó con dos grupos de tercer grado de educación primaria de una escuela de la cabecera municipal de Saucillo, Chih. México. Aunque la institución se encuentra en el centro de la población, el entorno inmediato del pueblo es rural. Los niños participantes son 54; 31 hombres y 23 mujeres, cuyas edades fluctúan entre los 8 y 9 años. 


\section{Instrumentos}

Se realizaron análisis descriptivos de la comprensión lectora integral y sus aspectos o rasgos específicos: fluidez, velocidad, identificación de la idea principal de un texto, localización de la información específica de un texto y utilización de información contenida en un texto para desarrollar un argumento. Con la suma de los puntajes de cada aspecto se construyó la variable independiente principal, comprensión lectora integral, como se indica en la tabla 2.

La definición operacional de las variables se presenta la tabla 2, en ella se explicita cómo se mide cada una de ellas, incluye dimensiones e indicadores de medida, con base en lo que propone la Secretaría de educación pública (SEP; 2011 y 2011) y Pinzás (1997). Los niveles de comprensión lectora, además de considerarse como puntaje valorativo (de 1 a 20 puntos) también se equiparó a los cuatro niveles de logro de las demás variables requiere apoyo, se acerca al estándar, estándar y avanzado, para efectos de exposición de análisis descriptivo-comparativo.

Para este estudio en particular se utilizaron las técnicas de encuesta y observación. Estas técnicas ayudaron al investigar el contexto en el que se desarrollan los alumnos, sus actitudes y habilidades de manera individual y grupal.

El planteamiento metodológico de investigación combina la encuesta, en un cuestionario, y la observación clínica, que interpreta lo que lee el sujeto, con base en criterios establecidos en la tabla 2. Tanto las respuestas al cuestionario como los datos de las observaciones realizadas son convertidas a niveles y puntajes, para ser procesados estadísticamente. Esta acción es complementada epistemológicamente con un análisis crítico de los resultados, en discusión basada en la comparación de resultados de otras investigaciones y de la pertinencia de los mismos en el contexto de la problemática de la investigación científica.

Tabla 2: Nivel de logro de comprensión lectora y forma de medición de rendimiento académico

\begin{tabular}{|c|c|c|c|}
\hline $\begin{array}{l}\text { Rasgo } \\
\text { evaluado }\end{array}$ & Nivel de logro & Rasgos & Puntaje \\
\hline \multirow{4}{*}{$\begin{array}{l}\text { Comprensión } \\
\text { de lectura }\end{array}$} & $\begin{array}{l}\text { Requiere } \\
\text { apoyo }\end{array}$ & $\begin{array}{l}\text { En este nivel el alumno sólo recupera algunos de los fragmentos del } \\
\text { texto, sin establecer conexión entre ellos }\end{array}$ & 1 \\
\hline & $\begin{array}{l}\text { Se acerca al } \\
\text { estándar }\end{array}$ & $\begin{array}{l}\text { El alumno recuerda alguno de los aspectos del texto, pero no localiza la } \\
\text { idea principal, ni el problema de la trama, tiene una idea general de lo } \\
\text { que trató este mismo, pero no logra organizar sus ideas para expresarse }\end{array}$ & 2 \\
\hline & Estándar & $\begin{array}{l}\text { En este nivel el alumno puede mencionar lo ocurrido en la narración, su } \\
\text { idea principal, los problemas y las soluciones que se le dio a éste, es } \\
\text { capaz de relatar los sucesos tal cual fueron. En este nivel el alumno aun } \\
\text { no alcanza a expresar lo que dedujo con fluidez }\end{array}$ & 3 \\
\hline & Avanzado & $\begin{array}{l}\text { En este nivel se recobra la información más importante del texto esto } \\
\text { incluye la idea principal, el problema y la solución de la narración, así } \\
\text { como sus personajes principales, además de esto, organiza los sucesos } \\
\text { tal y como sucedieron mencionando expresiones de tiempo }\end{array}$ & 4 \\
\hline \multirow{5}{*}{$\begin{array}{l}\text { Fluidez de } \\
\text { lectura }\end{array}$} & $\begin{array}{l}\text { Requiere } \\
\text { apoyo }\end{array}$ & $\begin{array}{l}\text { Los alumnos no son capases de leer una oración completa, esto provoca } \\
\text { que al final de la oración o el texto no puedan comprender la idea } \\
\text { principal }\end{array}$ & 1 \\
\hline & $\begin{array}{l}\text { Se acerca al } \\
\text { estándar }\end{array}$ & $\begin{array}{l}\text { Los estudiantes son capaces de leer dos o tres palabras seguidas, pero } \\
\text { no una oración completa, esto causa que no pueda entender a lo que se } \\
\text { relataba en el texto }\end{array}$ & 2 \\
\hline & & Son capaces de leer una ración completa sin pausas, pero no se respetan & \\
\hline & Estándar & $\begin{array}{l}\text { los signos de puntuación, aun así, el alumno puede rescatar a idea del } \\
\text { texto }\end{array}$ & 3 \\
\hline & Avanzado & $\begin{array}{l}\text { Los alumnos pueden leer textos completos, respetando signos de } \\
\text { puntuación y dándole la entonación adecuada al texto, es capaz de } \\
\text { comprender a lo que se refería el texto }\end{array}$ & 4 \\
\hline
\end{tabular}




\begin{tabular}{llll}
\hline \multirow{2}{*}{$\begin{array}{l}\text { Velocidad de } \\
\text { lectura }\end{array}$} & $\begin{array}{l}\text { Requiere } \\
\text { apoyo }\end{array}$ & Menor que 60 palabras por minuto & 1 \\
\cline { 2 - 4 } & $\begin{array}{l}\text { Se acerca al } \\
\text { estándar }\end{array}$ & De 60 a 84 palabras por minuto & 2 \\
\cline { 2 - 4 } & Estándar & De 85 a 99 palabras por minuto & 3 \\
\cline { 2 - 4 } & Avanzado & Mayor a 99 palabras por minuto & 4 \\
\hline \multirow{2}{*}{$\begin{array}{l}\text { Nivel de } \\
\text { comprensión } \\
\text { lectora }\end{array}$} & $\mathrm{n}$ & Sólo decodifica, pero no comprende lo que lee & 5 \\
\cline { 2 - 4 } & Inferencial & Comprende información implícita en el texto & 10 \\
\hline $\begin{array}{llll}\text { Puntaje de Comprensión lectora integral total } \\
\text { Rendimiento } \\
\text { académico }\end{array}$ & $\begin{array}{l}\text { Calificaciones } \\
\text { de exámenes }\end{array}$ & $\begin{array}{l}\text { Promedio de resultados de los test de conocimiento de la mesa técnica } \\
\text { educativa aplicados por bimestre }\end{array}$ & 20 \\
\hline
\end{tabular}

Fuente: SEP, (2011, 2012 y 2014) y Pinzás (1997). Adaptación propia

\section{Procedimiento}

Se utilizó como instrumento un cuestionario integrado por preguntas abiertas, autoadministrado de forma grupal, para facilitar su aplicación. Los cuestionamientos planteados se basan en los criterios establecidos en la tabla 2, en los aspectos de tipos y niveles de comprensión. Se evaluaron los niveles literal, inferencial y crítico de la comprensión lectora.

La observación, se basó en una interpretación de la lectura en términos de palabras leídas por minuto y comparadas con los criterios de la tabla 2 para establecer la velocidad de la lectura. Para ello a los alumnos se les hizo leer un texto, registrando el número de palabras leídas en el primer minuto. Para el caso de la fluidez, con base en la misma lectura, se clasificó a los estudiantes en los niveles correspondientes de la tabla 2. La lectura de referencia para la evaluación se titula: Los viajeros y el oso (Esopo, 2006).

En el procesamiento cuantitativo se realizaron análisis de correlación Rho de Spearman entre las variables de la comprensión lectora (fluidez, velocidad, identificación de la idea principal de un texto, localización de la información específica de un texto, tabla o gráfica, utiliza información contenida en un texto para desarrollar un argumento y la comprensión lectora integral) y el rendimiento académico, con pruebas bilaterales, tomando como referencia el nivel de alfa de .05 , como regla de decisión para determinar el rechazo o no de la hipótesis nula.

El paradigma de investigación es empírico analítico, toda vez que se basa en mediciones cuantitativas que se hace de las variables que participan en el estudio. Se considera que la construcción del conocimiento no puede ser absoluto, en cuanto al establecimiento de la verdad, por lo que siempre habrá un nivel de error, que debe ser mínimo, para llegar a conclusiones fundamentadas en un nivel de confianza aceptable (95\%).

Se utilizó el enfoque cuantitativo, con el fin de conseguir medidas de las variables y relaciones entre ellas, que permitan iniciar una discusión científica en el entendido de que ni el paradigma ni el enfoque eximen de la posibilidad de someter a un análisis crítico los resultados obtenidos por medio de la estadística descriptiva e inferencial.

El estudio es de tipo no experimental y transaccional o transversal, toda vez que se realiza en un solo momento, con análisis ex post facto, como lo plantean Hernández, Fernández y Baptista (2006). Se analizó la relación que existe entre la comprensión lectora y el rendimiento académico en los 54 alumnos, sin manipular ningún aspecto que influye en los resultados, los datos se recolectaron en una sola emisión, después del cuarto bimestre del ciclo escolar. La toma de 
información en tres bimestres anteriores sirvió de entrenamiento para la toma de lectura del cuarto bimestre.

Los alcances y limitaciones que tiene esta investigación están determinados por los grupos y el contexto en que se realiza el estudio. Los sujetos participantes no forman parte de una muestra representativa de una población más amplia. Los resultados no tienen el propósito de explicar más allá de lo que sucede a los dos grupos que intervienen en este proceso de indagación científica. En la discusión de los resultados se encuentran elementos que pudieran extrapolarse a otros contextos, que confirman otras investigaciones o que establecen discrepancias en los datos analizados y los de las referencias consultadas.

\section{Resultados}

Se analizaron las diferentes variables abordadas dentro de la investigación, las cuales fueron: velocidad lectora, fluidez lectora, identificar la idea principal en un texto, localizar información específica de un texto, utilizar información contenida en un texto para desarrollar un argumento y los niveles de comprensión lectora integral, esta última como variable integradora de todas las anteriores.

$\mathrm{Al}$ interpretar los datos relativos a la velocidad lectora, se observa que, en los dos grupos, el $55.5 \%$, de los alumnos, requieren apoyo, debido a que no alcanzan a leer más de 60 palabras por minuto, más del $30 \%$ se acercan al nivel estándar, ya que leen de 60 a 84 palabras por minuto, tres niños se encuentran en el nivel estándar que equivale a leer de 85 a 99 palabras por minuto y sólo dos alumnos se encuentran en el nivel avanzado.

Sobre fluidez lectora de los dos grupos, se interpreta que 15 alumnos requieren apoyo, 25 se acercan al nivel estándar, 13 se encuentran en el nivel estándar y sólo un alumno alcanzó en nivel avanzado. Estos resultados muestran que más del $50 \%$ de los alumnos no tienen el nivel estándar, se acerca al estándar, quiere decir que únicamente pueden leer frases cortas y no respetan los signos de puntuación, esto hace que pierdan la continuidad de la lectura y al final del texto no comprendan o recuerden la idea principal o datos importantes de éste.

Con respecto a la identificación de la idea principal de un texto, se identificó que cinco alumnos de los dos grupos logran identificar eficientemente la idea principal de un texto, diez alumnos se encuentran en el nivel estándar, dieciocho se acercan al estándar y veintiuno requieren ayuda, que es el nivel más bajo. Esto implica que estos alumnos únicamente son capaces de recordar pequeños fragmentos, pero no establecen ninguna conexión entre ellos. Estos resultados demuestran que la mayoría de los alumnos presentan dificultades para separar la información irrelevante de la importante que corresponde a la idea principal.

En lo que corresponde a la localización de información específica de un texto, se observa que la mayoría de los alumnos están por debajo del nivel estándar para su grado, ya que son 33 alumnos los que se encuentran en esta situación, 13 niños se encuentran en el nivel estándar y sólo cinco niños en el nivel avanzado.

Estos resultados dejan ver que la mayoría de los alumnos necesitan apoyo para obtener información de algún texto y no son capaces de realizarlo de una manera autónoma. Esto se convierte en un problema dentro del aula; primeramente, porque los niños no realizan actividades donde ellos busquen información por su cuenta y sinteticen la más importante, estas actividades son guiadas por el maestro, que tiene que indicar en qué parte se localiza la información relevante. 
En cuanto a la utilización de la información contenida en un texto para desarrollar un argumento, únicamente cuatro alumnos de los dos grupos alcanzan el nivel avanzado, nueve se encuentran en el nivel estándar, ocho se acercan al estándar y 33 alumnos requieren apoyo.

Los resultados de los niveles de comprensión lectora que se obtuvieron muestran que en el grupo de la sección A dos alumnos se encuentran en el nivel de decodificación, catorce en el nivel literal, cinco en el nivel inferencial y sólo tres en el nivel crítico; en el grupo B se identifica que cuatro alumnos se encuentran en el nivel de decodificación, dieciséis en el nivel literal, nueve en el nivel inferencial, y únicamente un estudiante en el nivel crítico.

Globalizando los resultados de ambos grupos, se observó que seis alumnos de los dos grupos aún se encuentran en el nivel de decodificación, treinta alumnos, que corresponden a más de la mitad de la población de estudio, se encuentran en el nivel literal, catorce en el nivel inferencial y sólo cuatro alumnos en el nivel crítico.

Continuando con resultados globales (ver figura 1), se encontró que el nivel más bajo, requiere apoyo, se observó en cuatro de las variables que se evaluaron: velocidad lectora, identificar la idea principal de un texto, localizar información específica y utilizar la información contenida en un texto. Este nivel fue el más frecuente en los datos observados. El segundo nivel con más frecuencia fue el denominado se acerca al estándar. El tercer nivel con más frecuencia fue el estándar. El cuarto nivel, el avanzado, se presenta menor frecuencia que los tres anteriores.

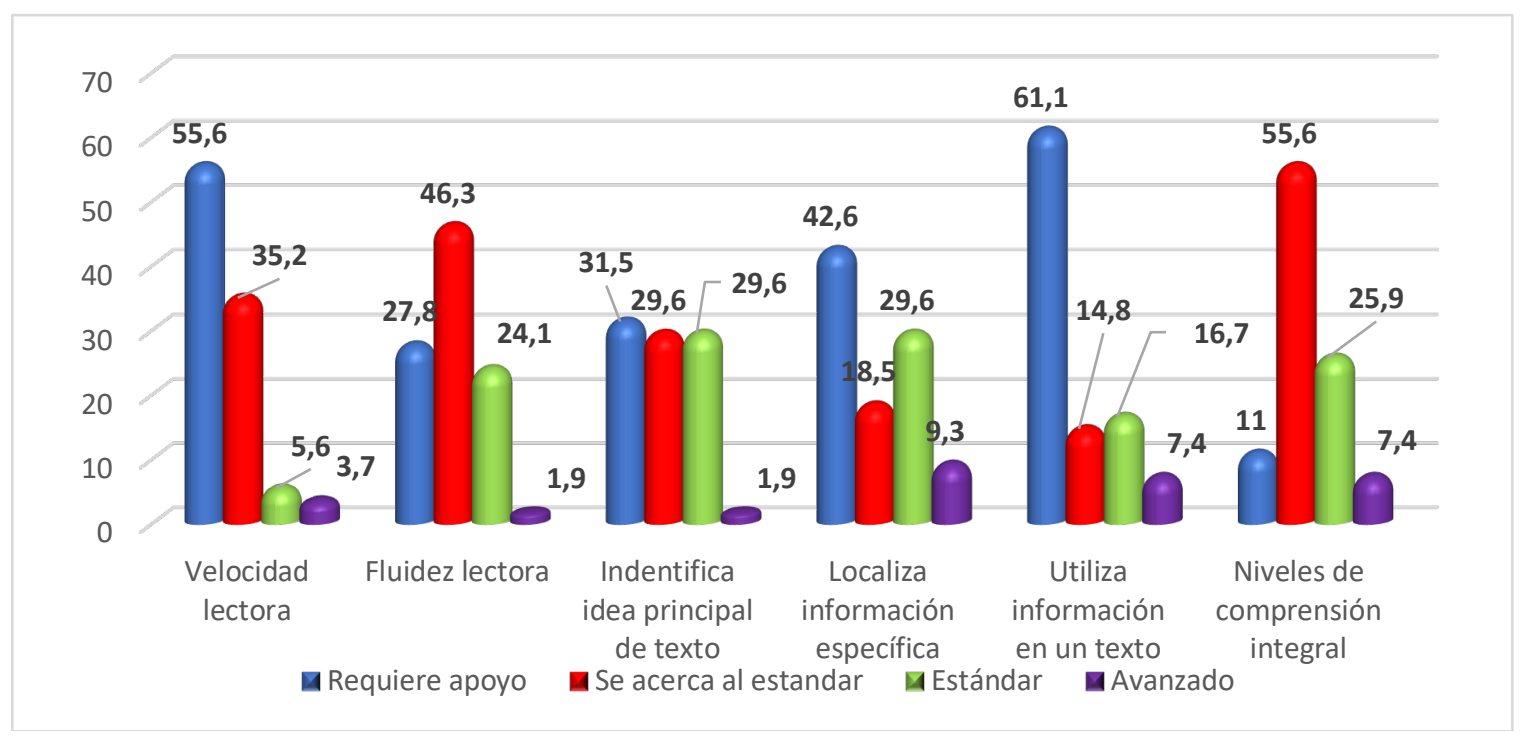

Figura 1. Resultados globales de comprensión lectora integral y sus aspectos específicos

De estos datos se infiere que de manera respectiva y sucesiva al más bajo nivel de comprensión le corresponde más niños, y viceversa, en el mayor nivel de lectura se encuentran menos sujetos evaluados. Esto refleja la problemática que existe en esos dos grupos de tercer grado de la educación primaria, que, dicho sea de paso, pertenecen a una de las escuelas con mejores resultados del municipio.

Para indagar sobre la relación existente entre los resultados de estos niveles de comprensión y el rendimiento académico, se efectuó el análisis correlacional Rho de Spearman. La regla de decisión que se aplicó se establece con un nivel de significancia de .05 para rechazar o no rechazar la hipótesis nula. 
En la tabla 3 se presentan los resultados obtenidos en las correlaciones entre el aprovechamiento académico de los niños y las variables: velocidad lectora, fluidez lectora, identificación de la idea principal, localiza información específica de un texto, utilizar información específica de un texto para desarrollar un argumento y la comprensión lectora integral.

Tabla 3. Resultado de prueba de hipótesis de correlaciones

\begin{tabular}{lllll}
\hline Variables & $\begin{array}{l}\text { Coeficiente } \\
\text { de } \\
\text { correlación }\end{array}$ & Sentido & $\begin{array}{l}\text { Sig. } \\
\text { (bilateral) }\end{array}$ & Decisión \\
\hline $\begin{array}{l}\text { Velocidad lectora versus Rendimiento } \\
\text { académico }\end{array}$ & $.665^{* *}$ & Positivo & .000 & Se rechaza la Ho \\
\hline Fluidez lectora versus promedio & $.654^{* *}$ & Positivo & .000 & Se rechaza la Ho \\
\hline $\begin{array}{l}\text { Identificar la idea principal del texto y } \\
\text { Rendimiento académico }\end{array}$ & $.476^{* *}$ & Positivo & .000 & Se rechaza la Ho \\
\hline $\begin{array}{l}\text { Localiza información específica de un texto, } \\
\text { tabla o gráfica versus Rendimiento académico }\end{array}$ & $.504^{* *}$ & Positivo & .000 & Se rechaza la Ho \\
\hline $\begin{array}{l}\text { Utilizar información específica de un texto } \\
\text { para desarrollar un argumento y Rendimiento } \\
\text { académico }\end{array}$ & $.489^{* *}$ & Positivo & .000 & Se rechaza la Ho \\
\hline $\begin{array}{l}\text { Comprensión lectora integral versus } \\
\text { Rendimiento académico }\end{array}$ & $.657^{* *}$ & Positivo & .000 & Se rechaza la Ho \\
\hline
\end{tabular}

En todas las pruebas se concluye que las variables relacionadas con la comprensión lectora se correlacionaron positivamente con el rendimiento académico de los niños, lo que significa que entre mayor puntaje se tenga en las evaluaciones de lectura mayor calificación promedio se tiene en el conjunto de materias de tercer grado de la escuela primaria.

La fuerza de las correlaciones se encuentra entre media y media alta. La más alta corresponde a la variable velocidad lectora, seguida por la fluidez, mientras que la más baja es la que implica la identificación de la idea principal de un texto. La fuerza general de correlaciones es aceptable en todos los casos.

Los niveles de significatividad que presentaron la correlaciones son menores que .001**, por lo que se toma la decisión de rechazar las hipótesis nulas que se plantean en cada caso de correlación, toda vez que el valor de alfa es menor al establecido en la regla de decisión (.05). En la tabla 4, se presentan los resultados obtenidos en la prueba de t de student para muestras independientes sobre la diferencia entre el grupo 1 y el grupo 2, con respecto a los elementos evaluados, relacionados con la comprensión lectora. Se realizó esto con la finalidad de comparar las mismas variables en los dos grupos para identificar si existen diferencias en los mismos, además de verificar si los instrumentos dan resultados similares en ambos grupos, para establecer su consistencia y validez de los mismos, considerando que los grupos son similares. 
Tabla 4. Prueba de $t$ de student, diferencia del grupo 1 y grupo 2

\begin{tabular}{llll}
\hline Variables & $\begin{array}{l}\text { Prueba T para la } \\
\text { igualdad de medias }\end{array}$ & $\begin{array}{l}\text { Sig. } \\
\text { (bilateral) }\end{array}$ & Decisión \\
\hline Velocidad lectora & -.275 & .784 & No se rechaza Ho \\
\hline Fluidez lectora & -.1 .799 & .078 & No se rechaza Ho \\
\hline Identifica la idea principal de un texto & 1.402 & .167 & No se rechaza Ho \\
\hline $\begin{array}{l}\text { Localiza la información específica de } \\
\text { un texto, tabla o grafica }\end{array}$ & -.602 & .549 & No se rechaza Ho \\
\hline $\begin{array}{l}\text { Utiliza información contenida en un } \\
\text { texto para desarrollar un argumento }\end{array}$ & .848 & .401 & No se rechaza Ho \\
\hline Comprensión lectora integral & .000 & 1.00 & No se rechaza Ho \\
\hline
\end{tabular}

En este análisis comparativo entre el grupo 1 y el grupo 2 con las variables que componen la comprensión lectora, se encontró que no existe diferencia significativa en ninguna de estas variables, ya que en todo su nivel de significancia supera el establecido $(p=.05)$. Esto indica que son grupos homogéneos entre sí, toda vez que las variables relacionadas con la comprensión lectora se comportan de manera similar en ambos grupos investigados.

Con respecto al sexo de los estudiantes y su asociación con la comprensión lectora integral y sus aspectos específicos, se realizó en análisis descriptivo, resultando ligeramente más altos los puntajes de los hombres en las variables velocidad de la lectura y fluidez. Por su parte, las mujeres tuvieron puntajes más altos en la identificación de la idea principal de un texto, en la localización de la información específica, en la utilización de la información contenida en un texto y en la comprensión lectora integral (tabla 5). También se identifican que las desviaciones estándares de las variables son más amplias en los promedios de género más altos, por ejemplo, en la velocidad lectora la media de los hombres es mucho más alta que la de las mujeres, pero la desviación de los datos es de 5.53, lo que lo hace inconsistente para ser significativa dicha diferencia, como se corrobora en la tabla 6 con las pruebas de hipótesis.

Tabla 5. Información estadística descriptiva organizada por sexo

\begin{tabular}{|c|c|c|c|c|c|}
\hline Variable de prueba & Sexo & $\mathbf{N}$ & Media & $\begin{array}{l}\text { Desv. } \\
\text { Desviación }\end{array}$ & $\begin{array}{l}\text { Desv. Error } \\
\text { promedio }\end{array}$ \\
\hline \multirow{2}{*}{$\begin{array}{l}\text { Palabra por minuto } \\
\text { (velocidad de la lectura) }\end{array}$} & Hombre & 31 & 59.71 & 30.821 & 5.536 \\
\hline & Mujer & 23 & 53.61 & 16.467 & 3.434 \\
\hline \multirow{2}{*}{ Fluidez lectora } & Hombre & 31 & 2.03 & .836 & .150 \\
\hline & Mujer & 23 & 1.96 & .706 & .147 \\
\hline \multirow{2}{*}{$\begin{array}{l}\text { Identificar la idea principal de un } \\
\text { texto }\end{array}$} & Hombre & 31 & 2.161290323 & 1.003220620 & .180183743 \\
\hline & Mujer & 23 & 2.173913043 & .984062725 & .205191262 \\
\hline \multirow{2}{*}{$\begin{array}{l}\text { Localizar la información específica } \\
\text { de un texto, tabla o gráfica }\end{array}$} & Hombre & 31 & 1.935483871 & .963863195 & .173114940 \\
\hline & Mujer & 23 & 2.217391304 & 1.166054799 & .243139233 \\
\hline \multirow{2}{*}{$\begin{array}{l}\text { Utiliza información contenida en un } \\
\text { texto para desarrollar un argumento. }\end{array}$} & Hombre & 31 & 1.612903226 & .919326542 & .165115921 \\
\hline & Mujer & 23 & 1.826086957 & 1.114049693 & .232295419 \\
\hline \multirow{2}{*}{ Comprensión lectora integral } & Hombre & 31 & 9.48 & 3.577 & .642 \\
\hline & Mujer & 23 & 9.52 & 3.666 & .765 \\
\hline
\end{tabular}

Estas diferencias con relación al sexo de los alumnos y las variables comprensión lectora, se según la prueba de significancia mediante el análisis de $t$ de student para muestras 
independientes, se encontró que no existe diferencia significativa en ninguna de estas variables, ya que todo nivel de significancia supera la $p=.05$, establecida en la regla de decisión, por los que se no se rechazan las hipótesis nulas. Esto indica que esta variable, sexo o género del estudiante, no se relaciona con comprensión lectora en la población utilizada. En la tabla 6 se presentan los resultados obtenidos en las pruebas de hipótesis.

Tabla 6. Prueba de t de student, diferencia sexo femenino y masculino

\begin{tabular}{llll}
\hline Variables & $\begin{array}{l}\text { Prueba T para la } \\
\text { igualdad de medias }\end{array}$ & $\begin{array}{l}\text { Sig. } \\
\text { (bilateral) }\end{array}$ & Decisión \\
\hline Comprensión lectora & -.38 & .970 & No se rechaza Ho \\
\hline Velocidad lectora & 1.913 & .061 & No se rechaza Ho \\
\hline Fluidez lectora & .351 & .727 & No se rechaza Ho \\
\hline Identifica la idea principal de un texto & -.046 & .963 & No se rechaza Ho \\
\hline $\begin{array}{l}\text { Localiza la información específica de un texto, } \\
\text { tabla o gráfica }\end{array}$ & -.972 & .336 & No se rechaza Ho \\
\hline $\begin{array}{l}\text { Utiliza información contenida en un texto para } \\
\text { desarrollar un argumento }\end{array}$ & -.770 & .445 & No se rechaza Ho \\
\hline
\end{tabular}

Estos resultados presentan evidencia que contradice la hipótesis de que alguno de los dos géneros, hombre o mujer, se ostente como el que tenga una mayor tendencia a obtener mejores puntajes en las evaluaciones de la comprensión lectora, por lo menos en lo que respecta a los grupos estudiados.

\section{Discusión y conclusiones}

Es importante señalar, que los resultados de esta investigación, representan únicamente a los alumnos participantes en la misma. No existe la intención de explicar o inferir que lo que se observó aplique para cualquier contexto o grupo, por respeto al planteamiento metodológico y a los alcances del proceso investigativo; sin embargo, es necesario comentar que se identificaron coincidencias significativas con lo que establece la literatura existente sobre este objeto de estudio. Lo que implica que existe una consistencia metodológica aceptable que permite visualizar lo que en otros contextos se ha observado mediante metodologías diversificadas.

La comprensión lectora en lo general, así como los elementos que se involucraron en la investigación, como la fluidez, velocidad, el nivel o tipo de comprensión lectora, y demás variables implicadas en esta investigación, son indicadores académicos importantes para quienes se encuentran transitando por instancias escolares o educativos formales, no formales e informales, toda vez que se convierten en herramientas con las que se apoyan para el desarrollo de los procesos individuales y sociales de la comunicación.

En la investigación se establece que existe correlación entre el rendimiento académico de los estudiantes y las variables de la comprensión lectora integral: velocidad lectora, fluidez lectora, identificación de la idea principal del texto, localización información específica de un texto y la utilización de la información contenida en un texto para desarrollar un argumento.

Los resultados señalan que los alumnos que no alcanzan un nivel adecuado para su grado, en alguno de los factores que intervienen en la comprensión lectora, tienen un rendimiento académico bajo, el tipo de lectura que realizan afecta el aprendizaje y provoca, en la mayoría de los casos, un rezago en los escolares. Estos datos son parecidos a los encontrados en la literatura (Aliaga, 2012; Gómez-Palomino, 2011; Llorens, 2015; García, 2018 y Solano, Manzanal y 
Jiménez, 2016), quienes encontraron relación significativa entre la comprensión lectora y rendimiento académico. Estos últimos explican que los alumnos que tienen mayor comprensión lectora utilizan más estrategias para comprender lo que leen; estas estrategias les ayudan a recuperar la información más importante del texto, así como a crear un pensamiento crítico.

Los resultados sobre la correlación de la fluidez lectora y el rendimiento académico, son similares a los encontrados por Calero (2016) y Gómez (2008), que señalan que la fluidez se debe tomar como un proceso simultáneo a la comprensión. Esta característica de la lectura es el reflejo del proceso que el lector realiza, que indica una verdadera interacción con el texto y su contenido, implicando, además, un nivel de comprensión aceptable que el que permita al lector que fluya la lectura de manera natural, y relacionar lo escrito con el patrón de sentido que le va dando en cada momento a la misma. Las personas que ya cuentan con esta capacidad, tienen ventajas significativas sobre los que aún no la tienen, proyectándose hacia un mayor éxito en las actividades académicas en lo general y hacia una mejor competencia comunicativa en los distintos ámbitos que le exige el contexto en el que se desenvuelven.

Con base en lo anterior se considera necesario profundizar en el estudio de la fluidez en el sentido en que los plantean Hudson, et al (2012) y Fumagalli, Barreyro y Jaichenco (2017), con la intención de establecer formas de evaluación más adecuadas de la comprensión lectora; así como la inclusión de otros referentes que propone el INEE (2012) para identificar niveles de comprensión en cuanto a tres procesos denominados: Acceder y recuperar, Integrar e interpretar y Reflexionar y evaluar.

Lo encontrado con respecto a diferenciar el nivel de competencia lectora entre hombres y mujeres, estrictamente en lo relacionado con la comprensión, se establece, por medio del análisis de $t$ de student para muestras independientes, que no existe una diferencia significativa entre ambos géneros, aunque el promedio de las mujeres (9.52) fue mayor al de los hombres (9.48), con una diferencia de 4 centésimas de punto. Por otra parte, existen resultados, como el de Ostria, et al (2014), quienes en el análisis descriptivo también encontraron mayor promedio de puntaje de competencia en las mujeres (3.12) que en los hombres (3.00), con una diferencia de 12 centésimas. Esta diferencia no fue probada con respecto a su significatividad, como se hizo en el presente estudio, por lo tanto, existe la duda si las diferencias establecidas por los promedios de ambos géneros son estadísticamente significativas o no.

Los resultados de evaluación de Lenguaje y comunicación realizado en México por el Instituto nacional de evaluación educativa en 2017 (INEE, 2018), también confirman esta tendencia hacia la identificación de resultados en el nivel insuficiente en las mujeres $(28.3 \%)$ que en los hombres (39.3\%).

Al analizar los índices de las correlaciones efectuadas, se destaca que la comprensión lectora se encuentra estrechamente relacionada con el rendimiento académico. Esto aporta evidencia de que la lectura es una variable importante en el aprovechamiento escolar de la población en la que se basó la investigación y que, según los resultados de esta y las investigaciones revisadas en la literatura.

Como cierre reflexivo y propositivo se asume que la evaluación de la comprensión lectora no tiene sentido si se utiliza como un fin en sí misma. Es decir, si sólo se evalúa para tener el dato de un nivel alcanzado o una calificación, de esa manera pierde su sentido didáctico como advierten Viana, et al (2014). La evaluación de la lectura debe aportar insumos que provoquen al docente a actuar en consecuencia de los resultados obtenidos y de la información general que aporta, cuantitativa y cualitativa, para mejorar los aprendizajes de los estudiantes. Una forma de hacerlo 
implica, como se señala en INEE (2012), considerar el uso de diversidad de textos, géneros y contextos comunicativos, con una variedad de propósitos conscientemente establecidos, otorgando sentido al texto, en situaciones de comunicación que incluyan y animen a los alumnos a incursionar en actos de lectura que le aporten la información necesaria para su vida y, si es posible, para hacer esta actividad más placentera.

\section{Referencias}

Aguillón, C. (2014). El proceso de comprensión lectora en niños escolares de 11 años. Zaragoza: México.

Aliaga, L. Y. (2012). Comprensión lectora y rendimiento académico en comunicación de alumnos del segundo grado de una institución educativa de ventanilla. Universidad San Ignacio De Loyola: Lima. Tesis de grado de maestría

Calero, A. (2014). Fluidez Lectora y Evaluación Formativa. Investigaciones sobre Lectura, 1, 33 48.

Casanova, M. A. (1998). La evaluación educativa Escuela básica. México: Muralla

Condemarín, M. (1989) Lectura temprana: jardín infantil y primer grado. Santiago de Chile: Andrés Bello.

Esopo, s/n. (2006) "Los viajeros y el oso" en Jerry Pinkney (adaptación), Fábulas de Esopo. México, SEP-Vicens Vives-Limusa,

Garbanzo, G. M. (2007). Factores asociados al rendimiento académico en estudiantes universitarios, una reflexión desde la calidad de la educación superior pública. Revista Educación, 31 (1), 43-63.

García, M. A.; Arévalo D., M. A. y Hernández, C. A. (2018). La comprensión lectora y el rendimiento escolar. Cuadernos de Lingüística Hispánica, 32, 155-174. Disponible en: http://www.scielo.org.co/pdf/clin/n32/2346-1829-clin-32-155.pdf

Gómez, L. F. (2008). El desarrollo de la competencia lectora en los primeros grados de primaria. Revista Latinoamericana de Estudios Educativos (México), XXXVIII (3-4), 95-126. Disponible en: http://www.redalyc.org/pdf/270/27012440005.pdf

Gómez-Palomino, J. (2011). Comprensión lectora y rendimiento escolar una ruta para mejorar la comunicación. COMUNI@CCIÓN: Revista de Investigación en Comunicación y Desarrollo, 2(2), 27-36.

Goodman, K. (1996) La lectura y los textos escritos: una perspectiva transaccional socio psicolingǘstica, Textos en Contextos 2. Los procesos de la lectura y la escritura. Argentina: Lectura y vida.

Gutierrez-Braojos, C., \& Salmerón P. H. (2012). Estrategias de comprensión lectora: enseñanza y evaluación en educación primaria. Profesorado. Revista de Currículum y Formación de Profesorado, 16 (1), 183-202. Disponible en: http://www.redalyc.org/pdf/567/56724377011.pdf

Hernández, H. M. (2007). Mecanismos para regular los distintos ritmos de aprendizaje en la comprensión lectora en alumnos de tercer grado de primaria. Sinaloa, México: Universidad Pedagógica Nacional

Hernández, R., Fernández, C. y Baptista, P. (2006). Metodología de la investigación. México: Mc Graw Hill/ Interamericana editores.

Hudson, R. F., Torgesen, J. K., Lane, B. y Turner, S. J. (2012). Relations among reading skills and sub-skills and text-level reading proficiency in developing readers. Reading and Writing: An Interdisciplinary Journal, 25(2), 483-507. 
INEE (2018). Panorama educativo de méxico. Indicadores del Sistema Educativo Nacional 2017. Educación básica y media superior. México: Instituto nacional para la evaluación de la educación.

Disponible

en:

http://publicaciones.inee.edu.mx/buscadorPub/P1/B/116/P1B116.pdf

INEE (2012). Textos continuos ¿Cómo se leen? La competencia lectora desde PISA. México: INEE. Disponible en: https://www.inee.edu.mx/wpcontent/uploads/2018/12/Textos_continuos.pdf

Jiménez-Pérez, E. (2014). Comprensión lectora VS Competencia lectora: qué son y qué relación existe entre ellas. Investigaciones Sobre Lectura, 1, 65-74.

Llorens, E. R. (2015). La comprensión lectora en Educación Primaria: Importancia e Influencia en los resultados académicos. Tesis de la Universidad Internacional de La Rioja. Disponible en: https://reunir.unir.net/bitstream/handle/123456789/3411/LLORENS\%20ESTEVE\%2C\% 20RUBEN.pdf? sequence $=1$

Madero, I. P. y Gómez, L. F. (2013). El proceso de comprensión lectora en alumnos de tercero de secundaria. Revista mexicana de investigación educativa, 18(56), 113-139.

OCDE (Organización para la Cooperación y el Desarrollo Económicos) (2006). La competencia lectora. PISA 2006. Marco de la evaluación. Conocimientos y habilidades en Ciencias, $\begin{array}{lllll}\text { Matemáticas } & y & \text { Lectura } & \text { Recuperado }\end{array}$ http://siie.tamaulipas.gob.mx/sistemas/docs/Pisa/comprension_lectora_PISA.pdf

OCDE (Organización para la Cooperación y el Desarrollo Económicos) (2012). Marcos y pruebas de evaluación de PISA 2012: matemáticas, lectura y ciencias. París: OCDE

Ostria, C. E., López M. G., Valenzuela, J.R. (2014). Caracterización de las competencias transversales. La competencia lectora: identificación del nivel de logro. Investigaciones Sobre Lectura, 2, 32-43.

Pinzás, G. J. (1997). Constructivismo y Aprendizaje de la Lectura. Lima: Pontificia Universidad Católica.

Ramos, G. Z. (2013). La comprensión lectora como una herramienta básica en la enseñanza de las ciencias naturales. Medellín, Colombia: Universidad Nacional de Colombia. Facultad de ciencias. Recuperado de: http://www.bdigital.unal.edu.co/11740/1/43731062.2014.pdf SEP (2011). Plan de estudios 2011. Educación básica. México: SEP

SEP (2012). Manual de procedimientos para el fomento y la valoración de la competencia lectora en el aular México: SEP. Recuperado de http://www.controlescolar.sep.gob.mx/work/models/controlescolar/Resource/carpeta_pdf /manual_fomento.pdf

SEP (2014). Estándares nacionales de habilidad lectora. Habilidad lectora. México: SEP. Recuperado de: http:/www.gob.mx/sep/acciones-y-programas/estandares-nacionales-dehabilidad-lectora-habilidad-lectora

SEP (2016). Modelo educativo 2016. México: SEP. Recuperado de: http://www.gob.mx/cms/uploads/attachment/file/118382/El_Modelo_Educativo_2016.pd f.

Silva, M. (2014). El estudio de la comprensión lectora en Latinoamérica: necesidad de un enfoque en la comprensión. Innovación Educativa. Vol.14, n.64, pp.47-55. Disponible en: http://www.scielo.org.mx/pdf/ie/v14n64/v14n64a5.pdf

Smith, F. (1989). Comprensión de la lectura. Análisis psicolingüístico de la lectura y su aprendizaje. México: Trillas.

Solano, N., Manzanal, A. I. y Jiménez, L. (2016). Estrategias de aprendizaje, comprensión lectora 
y rendimiento académico en Educación Secundaria, Psicología Escolar $e$ Educacional, 20(3), 447-456.

Solé, I. (2007). Estrategias de lectura/Reading Strategies. México: Advanced Marketing.

Solé, I. (2012). Competencia lectora y aprendizaje. Revista Iberoamericana de Educación, 59, 43-61.

Viana, F. L., Ribeiro, I. y Santos, S. (2014). Los desafíos de enseñar a comprender. Investigaciones Sobre Lectura, 1, 9-32. 


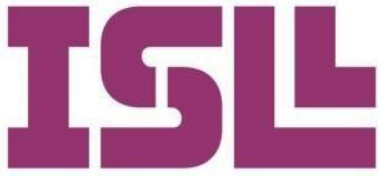

Number 12

December 2019

\title{
Reading comprehension and academic performance in Elementary Education
}

\author{
Efrén Viramontes Anaya \\ https://orcid.org/0000-0003-4378-3095 \\ Anahí Amparán Muro \\ https://orcid.org/0000-0003-0390-0883 \\ Luz Divina Núñez \\ Sifuentes \\ Escuela Normal Rural Ricardo Flores Magón
}

Recibido: 26-04-2018

Aceptado: 29-11-2019

Pag. 83 to 98

\section{Key words:}

Reading comprehension, academic performance, reading, reading fluency, reading speed.

\begin{abstract}
:
This research addresses the issue of interpret students' reading levels and types reading comprehension and the of blunders in it; there was a descriptive relationship with academic variable analysis, as well as the calculation performance in third-grade elementary of Spearman correlations and Student tstudents. The purpose is to assess the test for independent samples. The results links that both variables have and to indicate that comprehension is become aware of the importance of significantly correlated with academic reading in academic processes in achievement and that the condition of general. The study was developed in being male or female is not associated with two third-grade groups of a Mexican reading comprehension levels; however, elementary school, whose ages are there are coincidences with other between eight, and nine years old, the investigations that women present slightly socioeconomic level is average and the higher levels.

urban context is small, nestled in a rural area. The methodology was developed under a quantitative approach, interviewing and observation helped
\end{abstract}




\section{Introduction}

The research was carried out in a school in the north of Mexico, at the head of a rural municipality; it was carried out in two groups of third-grade primary education: the first of 26 students, 16 men and 10 women; the second of 28 students, 13 girls and 15 boys. All research subjects between the ages of 8 and 9 . The school is located in the center of the population of approximately 16000 habitants, surrounded by small rural communities that converge in this semiurban area for administrative purposes of the municipality, to attend the different schools or to purchase items they need for their homes and food. This community is deficient from the point of view of literacy, since there are not enough text carriers in a large city, nor enough reading acts in their homes, to have a good impact on children's literacy.

The argument for addressing this research topic focuses on the results of examinations by national and international agencies on children's reading comprehension in Mexican schools, as well as on assessments conducted at the local school level. To this, the importance of reading is added as a tool for cognitive analysis and learning of school and non-school contents that impact on the general education of the person.

In recent decades, reading comprehension in schools in most of the countries of the world has been evaluated very punctually, either by internal or external organizations in each country; derived from these evaluations, reading has been considered a priority matter of attention, mainly in basic education schools. In Mexico, the 2012 results of the Programme for International Student Assessment (PISA), (OECD, 2012) have been very low, regarding the other countries belonging to the OECD, recovering those results is not so simple, since it is established in that report that "if the current rates of improvement are maintained, it will take Mexico more than 25 years to reach the current OECD average levels in mathematics and more than 65 years in reading" (OECD, 2012, p. 3). These results are weighted in a just dimension by Silva (2014) when he points out that they are not simple figures, but they are a reflection of the educational realities of the countries evaluated, and that they imply a real educational problem that needs to be addressed.

In 2017, INEE (2018) applied the examination called PLANEA-ELSEN (National Plan for the Evaluation of Learning - Evaluation of Achievement referred to the National Education System), evaluating language development and communication, among other items, of which the results establish that there are $33.8 \%$ of secondary students who are in a deficient level, while only $26.1 \%$ of the population evaluated had satisfactory and outstanding scores. In these results, that show the lowest and highest levels, there is also a clear tendency towards the insufficiency of necessary elements in the development of language and communication; in this, reading comprehension has a fundamental role.

After analyzing the national and international results on the assessment of reading, this issue is considered to be crucial for training citizens in the field of literacy, in which writing and reading comprehension are fundamental elements. The development of literacy impacts on the school environment as well as on the daily life of the subject, which is why there is a need to improve children's reading from an early age.

For this reason, it is necessary to establish very specific forms of evaluation that can be applied by all elementary school teachers. It is assumed that reading comprehension is the essential part of students' academic development, since it becomes the main learning tool, as proposed by Solé (2012) and García, Arévalo \& Hernández (2017, p. 158), the latter express it textually: 
"reading in academic contexts has become more important and is called to participate more and more actively in the processes of continuous learning and learning for life". It is also warned that learning is affected by multiple factors, and that reading is only one of them. On these grounds, the main approach of this research arises with the question: what is the relationship between reading comprehension and the academic performance of students in the third grade of primary education?

The aim is to identify the relationship between these two variables; first, as a way of verifying what seems obvious, and also by establishing in a tangible way how the elements involved in reading comprehension behave with reference to students' academic achievement in primary school. The gender study in the results of the reading assessment is added to the analysis.

Some of the research that serves as a background to this study are: that of Aguillón (2014), with reference to the analysis of the lexical, semantic and syntactic elements of reading comprehension in 11 year old children, with quantitative methodology; the study that Solís (2014) made on reading comprehension and academic performance, analyzed the correlation between levels of reading comprehension and academic performance in the area of communication of students in second grade of primary education, in this study it was concluded that there is a significant positive relationship between the reading comprehension variable and performance in the area of communication; another study was that of Ramos (2013), on reading comprehension as a basic tool in the teaching of natural sciences, it was proposed to implement a strategy based on asking questions to improve the level of reading comprehension of scientific texts in the area of natural sciences, the result states that most students had improvement, in literal comprehension was where the largest population was located, then inferential and finally critical. Other references are the contributions of Madero \& Gómez (2011), on the process of reading comprehension in third of secondary school students and of Hernández (2007) about the rhythms of learning in reading comprehension in third-grade primary students.

One of the definitions of reading that serve as a conceptual reference is that offered by the $\operatorname{OECD}(2006$, p.48) that understands reading as "the ability to understand, use and analyze written texts, to reach the reader's aims, develop their knowledge and possibilities and participate in society", which is complemented with Gómez-Palomino's idea (2011, p. 28) when he points out that this activity "goes beyond the constructive production of the text, which is achieved under diverse endogenous and exogenous conditions", which implies an internal intellectual action of the subject and the interaction with the text, which is outside it, with specific, contextualized thematic information, expressed through specific signs. To synthesis, reading is considered as a communicative action between the writer and the reader. Internal action involves the cognitive sphere, from decoding, the identification of words in the text, the semantic meaning, as well as the comprehensive mental assignment coming from the contextualized experience of the subject reader.

As for reading comprehension, Gómez-Palomino (2011, p. 30) explains it as "a complex constructive activity of a strategic nature that involves the interactions of characteristics of the reader and of the text, within a given context", assigning the subject a very important role, since he has to construct the meaning of the text; between the visual and non-visual information raised by Smith (1989), between the text and the subject's frame of reference. This establishes a pattern of semantic comprehension that gives meaning to what is read, implying a series of intellectual actions, or strategies, proposed by Goodman (1996), that the reader puts into practice: sampling, prediction, inference, confirmation, and self-correction. 
The intention with which the reader approaches the text is a very important factor for comprehension since the motive acts as an information filter, in which what is sought is weighed and what is unimportant is discarded for this purpose.

The Ministry of Public Education of Mexico (SEP, 2011) divides the aspects that must be reached in terms of reading comprehension into cycles, in this case those that intervene in this research directly are those of the second cycle that involves the third and fourth grades of primary education, which are: Identify the main idea of a text, locate the specific information of a text and use information contained in a text to develop an argument.

In order to classify the level at which they are in each aspect, the SEP (2011) proposes four levels of achievement, which are used in basic education, for this reason, the aspects of each level can be adapted to the degree at which it is used. The established levels are: requires support, comes close to the standard, standard and advanced.

As for the types of reading comprehension, those proposed by Pinzás (1997) were taken as reference: decoding level, literal comprehension, inferential comprehension, and critical comprehension.

Reading fluency was also considered as part of comprehension, because it refers to the student's ability to read the text in a way that accesses and processes the information, respecting all punctuation marks as paralinguistic elements that provide content (SEP, 2011). Concerning the reading fluency construct, Fumagalli, Barreyro \& Jaichenco (2017) explain that when reading is done with a specific rhythm and speed that allows the reader to reconstruct the content of the text, and that when, for any reason, the reading loses that rhythm, the reconstruction of meanings is interrupted or lost. This depends, to a large extent, on a process that, according to these authors, is characterized by what they call strategies of decoding and automatic word recognition. They, like Hudson, Torgesen, Lane \& Turner (2012), consider speed, precision, and other aspects such as decoding and phonemic combination to be part of fluency.

For its part, the SEP (2011) proposes four levels to assess reading fluency: 1) require support, 2) Comes close to the standard, 3) standard and 4) advanced (Table 1), the latter level involves reading full texts, with punctuation and appropriate intonation, with the ability to understand the meaning of the text, and act accordingly or suggest something from it. This evaluation proposal is the one used in this research.

Table 2. Reading speed levels

\begin{tabular}{|c|c|c|c|c|}
\hline \multicolumn{5}{|c|}{ Elementary } \\
\hline School-level & $\begin{array}{l}\text { Require support } \\
\text { Level }\end{array}$ & $\begin{array}{l}\text { Come close to the } \\
\text { standard Level }\end{array}$ & Standard Level & Advanced Level \\
\hline First & Less than 15 & From 15 to 34 & From 35 to 59 & More than 59 \\
\hline Second & Less than 35 & From 35 to 59 & From 60 to 84 & More than 84 \\
\hline Third & Less than 60 & From 60 to 84 & From 85 to 99 & More than 99 \\
\hline Fourth & Less than 85 & From 85 to 99 & $\begin{array}{lll}\text { From } 100 \text { to } \\
114\end{array}$ & More than 114 \\
\hline Fifth & Less than 100 & From 100 to 114 & $\begin{array}{lll}\text { From } 115 \text { to } \\
124\end{array}$ & More than 124 \\
\hline Sixth & Less than 115 & From 115 to 124 & $\begin{array}{lll}\text { From } 125 \text { to } \\
134\end{array}$ & More than 134 \\
\hline
\end{tabular}

Source: SEP, 2012, p.11 
Reading speed, which also intervenes in comprehension, is considered as the number of words that can be read in a given time, it is evaluated independently, although it is linked to fluency. A too slow speed generally prevents comprehension, while an excessive speed would make such comprehension less possible, these limits depend on each subject, their reading skills, the text and their thematic frame of reference with which they face reading.

The measurement of this reading attribute is done directly by considering only the words per minute that are read by a person, as stated in SEP (2011). The fact of taking it into account to make a measurement of comprehension, is done only for the purpose of complementing the assessment of the variable called integral reading comprehension, and to attend the official suggestion of assessment of the Ministry of Public Education, not as a reflection of theoretical position that establishes the speed of reading as an independent element of fluency, since it agrees more with the idea of considering speed as an inherent part of fluency, as proposed by Hudson, et al (2012).

The process of reading comprehension is not a straight line, therefore, it requires taking into account other elements besides the speed of reading, such as performing reading regressions and applying autocorrections, this makes it take longer to read, but does not mean that the comprehension is less. Therefore, the assessment should include more elements in order to have a more integral idea of the process, such as those included in Table 2.

For the sole purpose of this research, the reading comprehension evaluation is considered as stated by Casanova (1998, p. 71): "obtaining rigorous and systematic information in order to have valid and reliable data close to a situation in order to form and make a valued judgment with respect to it", this refers to the fact that it should serve to improve educational practice, as well as to verify in which aspects learning processes can be optimized. It is a reference point for decisionmaking in the education field.

It is also important to consider, as Gutiérrez-Braojos \& Salmerón (2012) point out, that in order to carry out the reading assessment, the diversified use of information collection techniques is recommended in order to measure understanding. This makes it possible to carry out a triangulation of information that allows greater precision in the clinical and/or quantitative interpretation of the advances or levels in the comprehension of written texts.

The assumption that language teaching approaches have a very marked influence on the quality of students' reading comprehension is agreed upon, about this Solé (2007) explains two didactic processes in the construction of children's initial literacy, which affect the way in which reading comprehension is developed in their early stages; the first model, called bottom-up, is an ascendant reading method, in which it is considered that the reader first analyzes the letters, then the words in order to create a scheme that helps him to understand the text. It is considered ascending because it goes from the simplest to the most complex which is comprehension.

In this regard, Condemarín $(1989$, p. 75$)$ points out that "the most global skills are the identification of the word and the comprehension, which are divided into disaggregated units of contents and are associated with simple answers or classes of complex, automatic and precise answers", a reflection that coincides with what Solé (2007) stated in the top-down, descending or holistic model. This process is considered more appropriate than the first, because cognitive actions are carried out and the knowledge already possessed is applied, the writing is contextualized making significant connections with the text. These two ways of proceeding are defined in Llorens (2015) as analytical and synthetic procedures. There is a third model, more effective, named by Jiménez (2014) as interactive, in which complementary actions are carried 
out between the analytical (top-down) and synthetic (bottom-up) processes. This approach is taken as the assumption to develop quality comprehension in this research, founding the interaction on the social practices of language which is, considering reading within a communicative situation with real meaning and function.

Regarding the relationship between the sex or gender of students and the possibility that men or women may or may not be more likely to have a greater level of reading comprehension, research evidence was found, Ostria, López \& Valenzuela (2014) and the National Institute for Educational Evaluation (INEE, 2018), which indicates that women have better levels of comprehension than men. This gendered aspect is addressed in this study to provide more information in this regard, since the test of this hypothesis provides elements for teachers to consider it in their teaching strategies, with specific interventions needed.

The didactic attention for the development of reading comprehension in basic education children is supported by the scientific contributions of Viana, Ribeiro \& Santos (2014) and INEE (2012), the first in the sense and purpose that the evaluation of comprehension should have, to improve the educational processes of the students; the second, with reference to the concrete considerations of teaching in the subject of reading and the possibilities that one has to advance in comprehension.

It is established that the dependent variable of this research, academic performance, is an inserted factor in education, which is measured with the sum of the grades in which different factors intervene that determine the results of the students, and is defined as: "the quantified achievements that are reached in certain learning or subjects according to the study plan" (Garbanzo, 2007, p. 46).

\section{Objectives and hypotheses}

The main objectives of the research are twofold: first, to analyze the relationship between reading comprehension and reading indicators (fluency, speed, and localization of information) and the academic performance of the students; second, to establish whether there are differences between the sex of the students and the same reading indicators described above.

The Spearman Rho correlation hypotheses that were tested are the null version (bilateral analysis) of the following research hypotheses:

$\mathrm{H}_{1:}$ There is a relationship between reading fluency and the academic performance of third-grade students.

$\mathrm{H}_{2}$ : There is a relationship between the reading speed and the academic performance of third-grade students.

$\mathrm{H}_{3}$ : There is a relationship between the main idea identification of a text and the academic performance of third-grade students.

$\mathrm{H}_{4}$ : There is a relationship between the location of specific information in a text, chart, or graph and the academic performance of third-grade students.

$\mathrm{H}_{5}$ : There is a relationship between the use of the information contained in a text to develop an argument and the academic performance of third-grade students.

$\mathrm{H}_{6:}$ There is a relationship between integral reading comprehension and the academic performance of third-grade students. 
A Student t-test analysis was also performed for independent samples of the sex variable, concerning the scores of the variables that represent integral reading comprehension and its specific aspects. The decision rule is similar to that of the correlation tests $(p=.05)$.

In the student $t$-test analysis for independent samples that were tested by the null version (bilateral analysis) of the following research hypotheses:

$\mathrm{H}_{1:}$ There are significant differences between the sex of third-grade students and reading fluency.

$\mathrm{H}_{2}$ : There are significant differences between the sex of third-grade students and reading speed.

$\mathrm{H}_{3}$ : There are significant differences between the sex of third-grade students and the identification of the main idea of a text.

$\mathrm{H}_{4}$ : There are significant differences between the sex of third-grade students and the location of specific information in a text, chart, or graph.

$\mathrm{H}_{5:}$ There are significant differences between the sexes of third-grade students and the uses of information contained in a text to develop an argument.

$\mathrm{H}_{6:}$ There are significant differences between the sex of third-grade students and integral reading comprehension.

\section{Methodology}

\section{Participants}

The research was carried out with two groups of third-grade primary education from a school in the municipal capital of Saucillo, Chih. Mexico. Although the institution is in the center of the population, the immediate surroundings of the village are rural. The participating children are 54; 31 boys and 23 girls, whose ages fluctuate between 8 and 9 years.

\section{Instruments/Material}

Descriptive analyses of integral reading comprehension and its specific aspects or features were carried out: fluency, speed, identification of the main idea of a text, location of the specific information of a text and use of the information contained in a text to develop an argument. With the sum of the scores of each aspect, the main independent variable was constructed, integral reading comprehension, as indicated in table 2.

The operational definition of the variables is presented in Table 2, which explains how each of them is measured, including dimensions and measurement indicators, based on what is proposed by the Ministry of Public Education (SEP; 2011, 2012) and Pinzás (1997). The levels of reading comprehension, in addition to being considered as a value score (from 1 to 20 points) was also matched to the four levels of achievement of the other variables, requires support, is close to the standard, standard and advanced, for exposition purposes of descriptive-comparative analysis.

For this particular study, survey and observation techniques were used. These techniques helped by investigating the context in which students develop their attitudes and skills individually and in groups. 
The methodological exposition of research combines the survey, in a questionnaire, and the clinical observation, which interprets what the subject reads, based on criteria established in table 2. Both responses to the questionnaire and the data from the observations made are converted to levels and scores, to be processed statistically. This action is epistemologically complemented with a critical analysis of the results, in the discussion based on the comparison of the results of other researches and their pertinence in the context of the problematic of the scientific research.

Table 2: Reading comprehension achievement level and form of academic performance measurement

\begin{tabular}{|c|c|c|c|}
\hline $\begin{array}{l}\text { Rasgo } \\
\text { evaluado }\end{array}$ & Nivel de logro & Rasgos & Puntaje \\
\hline \multirow{4}{*}{$\begin{array}{l}\text { Comprensión } \\
\text { de lectura }\end{array}$} & $\begin{array}{l}\text { Requiere } \\
\text { apoyo }\end{array}$ & $\begin{array}{l}\text { En este nivel el alumno sólo recupera algunos de los fragmentos del } \\
\text { texto, sin establecer conexión entre ellos }\end{array}$ & 1 \\
\hline & $\begin{array}{l}\text { Se acerca al } \\
\text { estándar }\end{array}$ & $\begin{array}{l}\text { El alumno recuerda alguno de los aspectos del texto, pero no localiza la } \\
\text { idea principal, ni el problema de la trama, tiene una idea general de lo } \\
\text { que trató este mismo, pero no logra organizar sus ideas para expresarse }\end{array}$ & 2 \\
\hline & Estándar & $\begin{array}{l}\text { En este nivel el alumno puede mencionar lo ocurrido en la narración, su } \\
\text { idea principal, los problemas y las soluciones que se le dio a éste, es } \\
\text { capaz de relatar los sucesos tal cual fueron. En este nivel el alumno aun } \\
\text { no alcanza a expresar lo que dedujo con fluidez }\end{array}$ & 3 \\
\hline & Avanzado & $\begin{array}{l}\text { En este nivel se recobra la información más importante del texto esto } \\
\text { incluye la idea principal, el problema y la solución de la narración, así } \\
\text { como sus personajes principales, además de esto, organiza los sucesos } \\
\text { tal y como sucedieron mencionando expresiones de tiempo }\end{array}$ & 4 \\
\hline \multirow{4}{*}{$\begin{array}{l}\text { Fluidez de } \\
\text { lectura }\end{array}$} & $\begin{array}{l}\text { Requiere } \\
\text { apoyo }\end{array}$ & $\begin{array}{l}\text { Los alumnos no son capases de leer una oración completa, esto provoca } \\
\text { que al final de la oración o el texto no puedan comprender la idea } \\
\text { principal }\end{array}$ & 1 \\
\hline & $\begin{array}{l}\text { Se acerca al } \\
\text { estándar }\end{array}$ & $\begin{array}{l}\text { Los estudiantes son capaces de leer dos o tres palabras seguidas, pero } \\
\text { no una oración completa, esto causa que no pueda entender a lo que se } \\
\text { relataba en el texto }\end{array}$ & 2 \\
\hline & Estándar & $\begin{array}{l}\text { Son capaces de leer una ración completa sin pausas, pero no se respetan } \\
\text { los signos de puntuación, aun así, el alumno puede rescatar a idea del } \\
\text { texto }\end{array}$ & 3 \\
\hline & Avanzado & $\begin{array}{l}\text { Los alumnos pueden leer textos completos, respetando signos de } \\
\text { puntuación y dándole la entonación adecuada al texto, es capaz de } \\
\text { comprender a lo que se refería el texto }\end{array}$ & 4 \\
\hline \multirow{4}{*}{$\begin{array}{l}\text { Velocidad de } \\
\text { lectura }\end{array}$} & $\begin{array}{l}\text { Requiere } \\
\text { apoyo }\end{array}$ & Menor que 60 palabras por minuto & 1 \\
\hline & $\begin{array}{l}\text { Se acerca al } \\
\text { estándar }\end{array}$ & De 60 a 84 palabras por minuto & 2 \\
\hline & Estándar & De 85 a 99 palabras por minuto & 3 \\
\hline & Avanzado & Mayor a 99 palabras por minuto & 4 \\
\hline \multirow{4}{*}{$\begin{array}{l}\text { Nivel de } \\
\text { comprensión } \\
\text { lectora }\end{array}$} & $\begin{array}{l}\text { Decodificació } \\
\mathrm{n}\end{array}$ & Sólo decodifica, pero no comprende lo que lee & 5 \\
\hline & Literal & Decodifica y comprende lo que está explícitamente escrito & 10 \\
\hline & Inferencial & Comprende información implícita en el texto & 15 \\
\hline & Critica & $\begin{array}{l}\text { A partir de la comprensión de un texto propone, actúa y/o transforma la } \\
\text { realidad }\end{array}$ & 20 \\
\hline \multicolumn{4}{|c|}{ Puntaje de Comprensión lectora integral total } \\
\hline $\begin{array}{l}\text { Rendimiento } \\
\text { académico }\end{array}$ & $\begin{array}{l}\text { Calificaciones } \\
\text { de exámenes }\end{array}$ & $\begin{array}{l}\text { Promedio de resultados de los test de conocimiento de la mesa técnica } \\
\text { educativa aplicados por bimestre }\end{array}$ & $0-10$ \\
\hline
\end{tabular}

Source: SEP, (2011, 2012 y 2014) \& Pinzás (1997). Self-adaptation

\section{Procedure}

An open-ended questionnaire, self-administered in groups, was used as an instrument to facilitate its application. The questions presented are based on the criteria established in table 2, in the aspects of types and levels of comprehension. The literal, inferential and critical levels of 
reading comprehension were evaluated.

The observation was based on an interpretation of the reading in terms of words read per minute and compared with the criteria in table 2 to establish the speed of the reading. To do this, students were instructed to read a text, recording the number of words read in the first minute. In the case of fluency, based on the same reading, the students were classified in the corresponding levels of table 2. The reference reading for the assessment is entitled: Travellers and the Bear (Esopo, 2006).

In quantitative processing, Spearman's Rho correlation analysis was performed between the variables of reading comprehension (fluency, speed, identification of the main idea of a text, location of the specific information of a text, table or graph, uses information contained in a text to develop an argument and integral reading comprehension) and academic performance, with bilateral tests, taking as a reference the alpha level of .05, as a rule of decision to determine the rejection or not of the null hypothesis.

The research paradigm is empirical analytical since it is based on quantitative measurements made of the variables participating in the study. It is considered that the construction of knowledge cannot be absolute, in terms of establishing the truth, so there will always be a level of error, which must be minimal, to reach conclusions based on an acceptable level of confidence $(95 \%)$.

The research paradigm is empirical analytical, all the quantitative approach was used, in order to get measures of the variables and relationships between them, which allow initiating a scientific discussion in the understanding that neither the paradigm or the approach exempts from the possibility of subjecting to a critical analysis the results obtained by means of descriptive and inferential statistics.

The study is of a non-experimental and transactional or transversal type since it is carried out in a single moment, with ex post facto analysis, as proposed by Hernandez, Fernandez \& Baptista (2006). The relationship that exists between reading comprehension and academic performance in the 54 students were analyzed, without manipulating any aspect that influences the results; the data was collected in a single issue, after the fourth term of the school year cycle. The data collection in three previous bimesters served as training for the fourth bimester reading collection.

The reaches and limitations of this study are determined by the groups and the context in which the study is carried out. Participating subjects are not part of a representative sample of a larger population. The results are not intended to explain beyond what happens to the two groups involved in this process of scientific inquiry. In the discussion of the results, some elements could be extrapolated to other contexts, that confirm other investigations or to establish discrepancies in the analyzed data and those of the consulted references.

\section{Results}

The different variables addressed within the research were analyzed, which were: reading speed, reading fluency, identify the main idea in a text, locate specific information of a text, use information contained in a text to develop an argument and the levels of integral reading comprehension, the latter as a variable integrating all of the above.

When interpreting the data related to reading speed, it is observed that, in the two groups, $55.5 \%$ of the students require support, due to the fact that they cannot read more than 60 words 
per minute, more than $30 \%$ are close to the standard level, since they read from 60 to 84 words per minute, three children are in the standard level that is equivalent to reading from 85 to 99 words per minute and only two students are in the advanced level.

On reading fluency of the two groups, it is interpreted that 15 students require support, 25 students approach the standard level, 13 are at the standard level and only one student reached the advanced level. These results show that more than $50 \%$ of the students do not have the standard level, they are close to the standard, it means that they can only read short sentences and they do not respect the punctuation marks, this causes them to lose the continuity of the reading and at the end of the text they do not understand or remember the main idea or important data of this one.

Concerning the identification of the main idea of a text, it was identified that five students from the two groups efficiently identify the main idea of a text, ten students are at the standard level, eighteen students are close to the standard and twenty-one require help, which is the lowest level. This implies that these students are only able to remember small fragments, but do not establish any connection between them. These results show that most students have difficulty separating irrelevant information from important information corresponding to the main idea.

Regarding the location of specific information in a text, it is noticeable that the majority of students are below the standard level for their grade, since 33 students are in this situation, 13 children are in the standard level and only five children are in the advanced level.

These results show that the majority of students need to be supported to obtain information from some text and are not able to do it autonomously. This becomes a problem in the classroom; firstly, because children do not carry out activities where they look for information on their own and synthesize the most important information, these activities are guided by the teacher, who has to indicate where the relevant information is located.

Regarding the use of the information contained in a text to develop an argument, only four students from the two groups reach the advanced level, nine are at the standard level, eight are close to the standard, and 33 students require support.

The obtained results of the reading comprehension levels show that in the group of section A two students are in the decoding level, fourteen in the literal level, five in the inferential level and only three in the critical level; in group B it is determined that four students are in the decoding level, sixteen in the literal level, nine in the inferential level, and only one student in the critical level.

Globalizing the results of both groups, it was observed that six students from the two groups are still at the decoding level, thirty students, corresponding to more than half of the study population, are at the literal level, fourteen at the inferential level and only four students at the critical level.

Continuing with global results (see figure 1), it was found that the lowest level, requires support, was observed in four of the variables that were evaluated: reading speed, identifying the main idea of a text, locating specific information and using the information contained in a text. This level was the most frequent in the observed data. The second most frequent level was the one called "comes close to the standard". The third level most frequently was the standard. The fourth level, the advanced one, is less frequent than the three previous ones. 


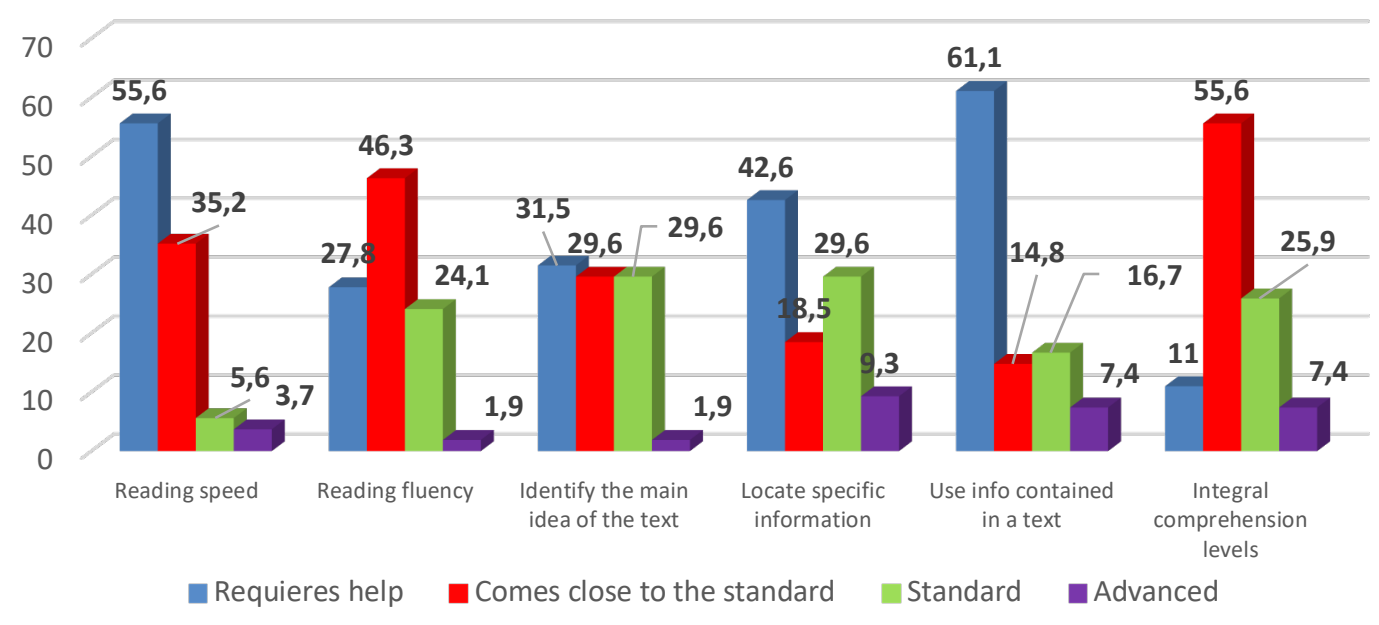

Figure 1. Overall results of integral reading comprehension and its specific aspects

From this data, it can be inferred that, respectively and successively, the lowest level of comprehension corresponds to more children, and vice versa, in the highest level of reading there are fewer subjects evaluated. This reflects the problems that exist in these two groups of thirdgrade primary education, which, incidentally, belong to one of the schools with the best results in the municipality.

To investigate the relationship between the results of these comprehension levels and academic performance, Spearman's Rho correlational analysis was performed. The decision rule that was applied is established with a significance level of .05 to reject or not the null hypothesis.

Table 3 presents the results obtained in the correlations between children's academic achievement and the variables: reading speed, reading fluency, identification of the main idea, localizing specific information from a text, using specific information from a text to develop an argument, and integral reading comprehension.

Table 3. Test result of correlation hypotheses

\begin{tabular}{lllll}
\hline Variables & $\begin{array}{l}\text { Correlation } \\
\text { coefficient }\end{array}$ & Sense & $\begin{array}{l}\text { Sig. } \\
\text { (bilateral) }\end{array}$ & Decision \\
\hline $\begin{array}{l}\text { Reading Speed versus Academic } \\
\text { Performance }\end{array}$ & $.665^{* *}$ & Positive & .000 & $\begin{array}{l}\text { The Ho is } \\
\text { rejected }\end{array}$ \\
\hline $\begin{array}{l}\text { Reading Fluency versus Average } \\
\text { Identify the main idea of the text } \\
\text { and Academic Performance }\end{array}$ & $.654^{* *}$ & Positive & .000 & $\begin{array}{l}\text { The Ho is } \\
\text { rejected }\end{array}$ \\
\hline $\begin{array}{l}\text { Locates specific information from a } \\
\text { text, chart, or graph versus } \\
\text { Academic Performance }\end{array}$ & $.504^{* *}$ & Positive & .000 & $\begin{array}{l}\text { The Ho is } \\
\text { rejected }\end{array}$ \\
\hline $\begin{array}{l}\text { Use text-specific information to } \\
\text { develop an argument and Academic } \\
\text { Performance }\end{array}$ & $.489^{* *}$ & Positive & .000 & $\begin{array}{l}\text { The Ho is } \\
\text { rejected }\end{array}$ \\
\hline $\begin{array}{l}\text { Integral Reading Comprehension } \\
\text { versus Academic Performance }\end{array}$ & $.657^{* *}$ & Positive & .000 & $\begin{array}{l}\text { The Ho is } \\
\text { rejected }\end{array}$ \\
\hline
\end{tabular}

In all the tests it is concluded that the variables related to reading comprehension were positively correlated with the academic performance of the children, which means that the higher 
the score on the reading assessments the higher the average grade is in the entire set of third-grade elementary school subjects.

The strength of the correlations lies between medium and medium-high. The highest corresponds to the variable reading speed, followed by fluency, while the lowest is that which implies the identification of the main idea of a text. The general strength of correlations is acceptable in all cases.

The levels of significance presented by the correlations are lower than $.001^{* *}$, so the decision is taken to reject the null hypotheses that arise in each case of correlation since the value of alpha is lower than that established in the decision rule (.05).

Table 4 presents the results obtained in the student t-test for independent samples on the difference between group 1 and group 2, with reference to the elements evaluated, related to reading comprehension. This was done to compare the same variables in the two groups to identify if there are differences in the same, as well as to verify if the instruments give similar results in both groups, to establish their consistency and validity, considering that the groups are similar.

Table 4. Student t-test, the difference of group 1 and group 2

\begin{tabular}{llll}
\hline Variables & $\begin{array}{l}\text { T-test for } \\
\text { equality of } \\
\text { averages }\end{array}$ & $\begin{array}{l}\text { Sig. } \\
\text { (bilateral) }\end{array}$ & Decision \\
\hline Reading speed & -.275 & .784 & $\begin{array}{c}\text { The Ho is not } \\
\text { rejected }\end{array}$ \\
\hline Reading fluency & -.1 .799 & .078 & $\begin{array}{l}\text { The Ho is not } \\
\text { rejected }\end{array}$ \\
\hline Identifies the main idea of a text & 1.402 & .167 & $\begin{array}{l}\text { The Ho is not } \\
\text { rejected }\end{array}$ \\
\hline $\begin{array}{l}\text { Locates specific information from } \\
\text { a text, chart, or graph }\end{array}$ & -.602 & .549 & $\begin{array}{l}\text { The Ho is not } \\
\text { rejected }\end{array}$ \\
\hline $\begin{array}{l}\text { Uses the information contained in } \\
\text { a text to develop an argument }\end{array}$ & .848 & .401 & $\begin{array}{l}\text { The Ho is not } \\
\text { rejected }\end{array}$ \\
\hline \begin{tabular}{l} 
Integral reading comprehension \\
\hline
\end{tabular} & .000 & 1.00 & $\begin{array}{l}\text { The Ho is not } \\
\text { rejected }\end{array}$ \\
\hline
\end{tabular}

In this comparative analysis between group 1 and group 2 with the variables that make up the reading comprehension, it was found that there is no significant difference in any of these variables since in all their level of significance exceeds the established $(p=.05)$. This indicates that they are homogeneous groups among themselves since the variables related to reading comprehension behave similarly in both investigated groups.

About the sex of the students and their association with integral reading comprehension and its specific aspects, it was carried out in descriptive analysis, resulting in slightly higher scores for men in the variables of reading speed and fluency. For their part, women scored higher in identifying the main idea of a text, in locating specific information, in using the information contained in a text, and in integral reading comprehension (table 5). It is also identified that the standard deviations of the variables are wider in higher gender averages, such as, for example, in the reading speed the average of men is much higher than that of women, but the deviation of the data is 5.53, which makes it inconsistent to be significant such difference, as is corroborated in Table 6 with hypothesis tests. 
Table 5. Descriptive statistical information organized by sex

\begin{tabular}{|c|c|c|c|c|c|}
\hline Test variable & Sex & $\mathbf{N}$ & Average & Div. Deviation & $\begin{array}{l}\text { Average error } \\
\text { deviation }\end{array}$ \\
\hline \multirow{2}{*}{$\begin{array}{l}\text { Word per minute } \\
\text { (reading speed) }\end{array}$} & Male & 31 & 59.71 & 30.821 & 5.536 \\
\hline & Female & 23 & 53.61 & 16.467 & 3.434 \\
\hline \multirow{2}{*}{ Reading fluency } & Male & 31 & 2.03 & .836 & .150 \\
\hline & Female & 23 & 1.96 & .706 & .147 \\
\hline \multirow{2}{*}{ Identify the main idea of the text } & Male & 31 & 2.161290323 & 1.003220620 & .180183743 \\
\hline & Female & 23 & 2.173913043 & .984062725 & .205191262 \\
\hline \multirow{2}{*}{$\begin{array}{l}\text { Locate specific information from a } \\
\text { text, chart, or graph }\end{array}$} & Male & 31 & 1.935483871 & .963863195 & .173114940 \\
\hline & Female & 23 & 2.217391304 & 1.166054799 & .243139233 \\
\hline \multirow{2}{*}{$\begin{array}{l}\text { Uses the information contained in a } \\
\text { text to develop an argument. }\end{array}$} & Male & 31 & 1.612903226 & .919326542 & .165115921 \\
\hline & Female & 23 & 1.826086957 & 1.114049693 & .232295419 \\
\hline \multirow{2}{*}{ Integral reading comprehension } & Male & 31 & 9.48 & 3.577 & .642 \\
\hline & Female & 23 & 9.52 & 3.666 & .765 \\
\hline
\end{tabular}

These differences in relation to the sex of the students and the reading comprehension variables, according to the test of significance by means of the analysis of student t-test for independent samples, showed that there is no significant difference in any of these variables, since every level of significance exceeds $p=.05$, established in the decision rule, by which null hypotheses are not rejected. This indicates that this variable, sex or gender of the student, is not related to reading comprehension in the population used. Table 6 presents the results obtained in the hypothesis tests.

Table 6. Student t-test, female and male sex difference

\begin{tabular}{llll}
\hline Variables & $\begin{array}{l}\text { T-test for equality } \\
\text { of averages }\end{array}$ & $\begin{array}{l}\text { Sig. } \\
\text { (bilateral) }\end{array}$ & Decision \\
\hline Reading comprehension & -.38 & .970 & $\begin{array}{l}\text { The Ho is not } \\
\text { rejected }\end{array}$ \\
\hline Reading speed & 1.913 & .061 & $\begin{array}{l}\text { The Ho is not } \\
\text { rejected }\end{array}$ \\
\hline Reading fluency & .351 & .727 & $\begin{array}{l}\text { The Ho is not } \\
\text { rejected }\end{array}$ \\
\hline Identifies the main idea of the text & -.046 & .963 & $\begin{array}{l}\text { The Ho is not } \\
\text { rejected }\end{array}$ \\
\hline $\begin{array}{l}\text { Locates specific information from a } \\
\text { text, chart, or graph }\end{array}$ & -.972 & .336 & $\begin{array}{l}\text { The Ho is not } \\
\text { rejected }\end{array}$ \\
\hline $\begin{array}{l}\text { Uses the information contained in a } \\
\text { text to develop an argument }\end{array}$ & -.770 & .445 & $\begin{array}{l}\text { The Ho is not } \\
\text { rejected }\end{array}$ \\
\hline
\end{tabular}

These results present evidence that contradicts the hypothesis that either gender, male or female, has the greatest tendency to score better on reading comprehension assessments, at least for the studied groups.

\section{Discussion and conclusions}

It is important to point out that the results of this research represent only the students participating in it. There is no intention of explaining or inferring that what was observed applies to any context or group, out of respect for the methodological approach and the scope of the 
research process; however, it is necessary to comment that significant coincidences were identified with what is established in the existing literature on this object of study. This implies that there is an acceptable methodological consistency that allows visualizing what in other contexts has been observed through diversified methodologies.

Reading comprehension in general, as well as the elements involved in the research, such as fluency, speed, the level or type of reading comprehension, and other variables involved in this research, are important academic indicators for those who are passing through formal, non-formal and informal school or educational instances, since they become tools with which they support the development of individual and social communication processes.

The research establishes that there is a correlation between the academic performance of students and the variables of integral reading comprehension: reading speed, reading fluency, identification of the main idea of the text, location of specific information of a text and the use of the information contained in a text to develop an argument.

The results indicate that students who do not reach an adequate level for their grade, in some of the factors that intervene in reading comprehension, have a low academic performance, the type of reading they do affects learning and causes, in most cases, an educational lag. This data is similar to those found in the literature (Aliaga, 2012; Gómez-Palomino, 2011; Llorens, 2015; García, 2018 and Solano, Manzanal \& Jiménez, 2016), who found a significant relationship between reading comprehension and academic performance. The latter explains that students with greater reading comprehension use more strategies to understand what they read; these strategies help them recover the most important information in the text, as well as create critical thinking.

The results on the correlation of reading fluency and academic performance are similar to those found by Calero (2016) and Gómez (2008), which indicate that fluency should be taken as a simultaneous process to comprehension. This characteristic of reading is the reflection of the process that the reader carries out, which indicates a true interaction with the text and its content, implying, in addition, an acceptable level of comprehension that allows the reader to flow the reading naturally, and to relate what is written with the pattern of meaning that gives it at each moment. People who already have this capacity have significant advantages over those who do not have it yet, projecting themselves towards greater success in academic activities in general and towards a better communicative competence in the different fields demanded by the context in which they operate.

Based on the above, it is considered necessary to deepen the study of fluidity in the sense that Hudson, et al (2012) and Fumagalli, Barreyro and Jaichenco (2017) suggest, with the intention of establishing more appropriate forms of evaluation of reading comprehension; as well as the inclusion of other references proposed by INEE (2012) to identify levels of understanding regarding three processes called: Access and recover, Integrate and interpret and Reflect and evaluate.

What was found in relation to distinguishing the level of reading competence between boys and girls, strictly in relation to comprehension, establishes, through the analysis of student t-test for independent samples, that there is no significant difference between both genders, although the average of women (9.52) was higher than that of men (9.48), with a difference of 4 hundredths of a point. On the other hand, there are results, such as Ostria et al (2014), who in the descriptive analysis also found a higher average score of competence in women (3.12) than in men (3.00), with a difference of 12 hundredths. This difference was not proven concerning its significance, as it was done in the present study, therefore, there is a doubt if the differences established by the 
averages of both genders are statistically significant or not.

The results of the evaluation of Language and Communication carried out in Mexico by the National Institute for Educational Evaluation in 2017 (INEE, 2018), also confirm this trend towards the identification of results at the insufficient level in women $(28.3 \%)$ than in men $(39.3$ $\%)$.

When analyzing the indexes of the correlations carried out, it is emphasized that reading comprehension is closely related to academic performance. This provides evidence that reading is an important variable in the educational attainment of the population on which the research was based and that, according to the results of this research and the research reviewed in the literature, reading is an important variable in the educational attainment of the population on which the research was based.

As a reflective and propositional closure, it is assumed that the evaluation of reading comprehension is meaningless if used as an end in itself. That is to say if it is only evaluated to have the data of a level reached or a grade, in this way it loses its didactic sense as Viana et al (2014) warn. The evaluation of reading should provide inputs that provoke the teacher to act as a consequence of the results obtained and of the general information it provides, both quantitative and qualitative, to improve student learning. One way of doing this implies, as stated in INEE (2012), considering the use of diversity of texts, genres and communicative contexts, with a variety of consciously established purposes, giving meaning to the text, in communication situations that include and encourage students to engage in acts of reading that provide the necessary information for their lives and, if possible, to make this activity more enjoyable.

\section{References}

Aguillón, C. (2014). El proceso de comprensión lectora en niños escolares de 11 años. Zaragoza: México.

Aliaga, L. Y. (2012). Comprensión lectora y rendimiento académico en comunicación de alumnos del segundo grado de una institución educativa de ventanilla. Universidad San Ignacio De Loyola: Lima. Tesis de grado de maestría

Calero, A. (2014). Fluidez Lectora y Evaluación Formativa. Investigaciones sobre Lectura, $1,33-48$.

Casanova, M. A. (1998). La evaluación educativa Escuela básica. México: Muralla.

Condemarín, M. (1989) Lectura temprana: jardín infantil y primer grado. Santiago de Chile: Andrés Bello.

Esopo, s/n. (2006) “Los viajeros y el oso” en Jerry Pinkney (adaptación), Fábulas de Esopo. México, SEP-Vicens Vives-Limusa,

Garbanzo, G. M. (2007). Factores asociados al rendimiento académico en estudiantes universitarios, una reflexión desde la calidad de la educación superior pública. Revista Educación, $31(1), 43-63$.

García, M. A., Arévalo, M. A., \& Hernández, C. A. (2018). La comprensión lectora y el rendimiento escolar. Cuadernos de Lingüística Hispánica, 32, 155-174. Disponible en: http://www.scielo.org.co/pdf/clin/n32/2346-1829-clin-32-155.pdf

Gómez, L. F. (2008). El desarrollo de la competencia lectora en los primeros grados de primaria. Revista Latinoamericana de Estudios Educativos (México), XXXVIII (3-4), 95-126. Disponible en: http://www.redalyc.org/pdf/270/27012440005.pdf

Gómez-Palomino, J. (2011). Comprensión lectora y rendimiento escolar una ruta para 
mejorar la comunicación. COMUNI@CCIÓN: Revista de Investigación en Comunicación y Desarrollo, 2(2), 27-36.

Goodman, K. (1996) La lectura y los textos escritos: una perspectiva transaccional socio psicolingüística, Textos en Contextos 2. Los procesos de la lectura y la escritura. Argentina: Lectura y vida.

Gutierrez-Braojos, C., \& Salmerón, P. H. (2012). Estrategias de comprensión lectora: enseñanza y evaluación en educación primaria. Profesorado. Revista de Currículum y Formación de Profesorado, 16 (1), 183-202. Disponible en: http://www.redalyc.org/pdf/567/56724377011.pdf

Hernández, H. M. (2007). Mecanismos para regular los distintos ritmos de aprendizaje en la comprensión lectora en alumnos de tercer grado de primaria. Sinaloa, México: Universidad Pedagógica Nacional

Hernández, R., Fernández, C., \& Baptista, P. (2006). Metodología de la investigación. México: Mc Graw Hill/ Interamericana editores.

Hudson, R. F., Torgesen, J. K., Lane, B., \& Turner, S. J. (2012). Relations among reading skills and sub-skills and text-level reading proficiency in developing readers. Reading and Writing: An Interdisciplinary Journal, 25(2), 483-507.

INEE (2018). Panorama educativo de méxico. Indicadores del Sistema Educativo Nacional 2017. Educación básica y media superior. México: Instituto nacional para la evaluación de la educación. Disponible en: http://publicaciones.inee.edu.mx/buscadorPub/P1/B/116/P1B116.pdf

INEE (2012). Textos continuos ¿Cómo se leen? La competencia lectora desde PISA. México: INEE. Disponible en: https://www.inee.edu.mx/wpcontent/uploads/2018/12/Textos_continuos.pdf

Jiménez-Pérez, E. (2014). Comprensión lectora VS Competencia lectora: qué son y qué relación existe entre ellas. Investigaciones Sobre Lectra, 1, 65-74.

Llorens, E. R. (2015). La comprensión lectora en Educación Primaria: Importancia e Influencia en los resultados académicos. Tesis de la Universidad Internacional de La Rioja. Disponible

en: https://reunir.unir.net/bitstream/handle/123456789/3411/LLORENS\%20ESTEVE\%2C\%20RUB EN.pdf?sequence $=1$

Madero, I. P., \& Gómez, L. F. (2013). El proceso de comprensión lectora en alumnos de tercero de secundaria. Revista mexicana de investigación educativa,18(56), 113-139.

OCDE (Organización para la Cooperación y el Desarrollo Económicos) (2006). La competencia lectora. PISA 2006. Marco de la evaluación. Conocimientos y habilidades en Ciencias, Matemáticas $\quad y \quad$ Lectura. Recuperado de http://siie.tamaulipas.gob.mx/sistemas/docs/Pisa/comprension_lectora_PISA.pdf

OCDE (Organización para la Cooperación y el Desarrollo Económicos) (2012). Marcos y pruebas de evaluación de PISA 2012: matemáticas, lectura y ciencias. París: OCDE

Ostria, C. E., López M. G., \& Valenzuela, J.R. (2014). Caracterización de las competencias transversales. La competencia lectora: identificación del nivel de logro. Investigaciones Sobre Lectura, 2, 32-43.

Pinzás, G. J. (1997). Constructivismo y Aprendizaje de la Lectura. Lima: Pontificia Universidad Católica.

Ramos, G. Z. (2013). La comprensión lectora como una herramienta básica en la enseñanza de las ciencias naturales. Medellín, Colombia: Universidad Nacional de Colombia. Facultad ciencias. 
http://www.bdigital.unal.edu.co/11740/1/43731062.2014.pdf

SEP (2011). Plan de estudios 2011. Educación básica. México: SEP

SEP (2012). Manual de procedimientos para el fomento y la valoración de la competencia lectora en el aula. México: SEP. Recuperado de http://www.controlescolar.sep.gob.mx/work/models/controlescolar/Resource/carpeta pdf/manua 1 fomento.pdf

SEP (2014). Estándares nacionales de habilidad lectora. Habilidad lectora. México: SEP. Recuperado de: http://www.gob.mx/sep/acciones-y-programas/estandares-nacionales-dehabilidad-lectora-habilidad-lectora

SEP (2016). Modelo educativo 2016. México: SEP. Recuperado de: http://www.gob.mx/cms/uploads/attachment/file/118382/El_Modelo_Educativo_2016.pdf.

Silva, M. (2014). El estudio de la comprensión lectora en Latinoamérica: necesidad de un enfoque en la comprensión. Innovación Educativa. Vol.14, n.64, pp.47-55. Disponible en: http://www.scielo.org.mx/pdf/ie/v14n64/v14n64a5.pdf

Smith, F. (1989). Comprensión de la lectura. Análisis psicolingüistico de la lectura y su aprendizaje. México: Trillas.

Solano, N., Manzanal, A. I., \& Jiménez, L. (2016). Estrategias de aprendizaje, comprensión lectora y rendimiento académico en Educación Secundaria, Psicología Escolar e Educacional, 20(3), 447-456.

Solé, I. (2007). Estrategias de lectura/Reading Strategies. México: Advanced Marketing.

Solé, I. (2012). Competencia lectora y aprendizaje. Revista Iberoamericana de Educación, 59, 43-61.

Viana, F. L., Ribeiro, I., \& Santos, S. (2014). Los desafíos de enseñar a comprender. Investigaciones Sobre Lectura, 1, 9-32. 